Draft Version March 15, 2019

Typeset using $\mathrm{LAT}_{\mathrm{E}} \mathrm{X}$ preprint2 style in AASTeX61

\title{
THE XMM-NEWTON/HST VIEW OF THE OBSCURING OUTFLOW IN THE SEYFERT GALAXY MRK 335 OBSERVED AT EXTREMELY LOW X-RAY FLUX.
}

\author{
Anna Lia Longinotti, ${ }^{1,2}$ Gerard Kriss, ${ }^{3}$ Yair Krongold,${ }^{4}$ Karla Z. Arellano-Cordova, ${ }^{1,5}, 6$ \\ Stefanie Komossa,${ }^{7}$ Luigi Gallo,${ }^{8}$ Dirk Grupe, ${ }^{9}$ Smita Mathur,${ }^{10}$ Michael Parker, ${ }^{11}$ \\ Anil Pradhan, ${ }^{10}$ And Dan Wilkins ${ }^{12}$
}

\footnotetext{
${ }^{1}$ Instituto Nacional de Astrofísica, Óptica y Electrónica, Luis E. Erro 1, Tonantzintla, Puebla, México, C.P. 72840

${ }^{2}$ CONACyT-INAOE

${ }^{3}$ Space Telescope Science Institute, 3700 S. Martin Drive, Baltimore, MD 21218, USA

${ }^{4}$ Instituto de Astronomía, Universidad Nacional Autónoma de México, Circuito Exterior, Ciudad Universitaria, Ciudad de México 04510, México

${ }^{5}$ Instituto de Astrofísica de Canarias, E-38200 La Laguna, Tenerife, Spain

${ }^{6}$ Departamento de Astrofísica, Universidad de La Laguna, E-38206 La Laguna, Tenerife, Spain

${ }^{7}$ Max Planck Institut fuer Radioastronomie, Auf dem Huegel 69,53121 Bonn, Germany

${ }^{8}$ Department of Astronomy and Physics, Saint Mary's University, Halifax, Canada

${ }^{9}$ Department of Earth and Space Sciences, Morehead State University, 235 Martindale Drive, Morehead, KY, USA

${ }^{10}$ Department of Astronomy, Ohio State University, 140 West 18th Avenue, Columbus, Ohio 43210-1173

${ }^{11}$ ESAC, PO Box 78, 28691 Villanueva de la Cañada, Madrid, Spain

${ }^{12}$ Kavli Institute for Particle Astrophysics and Cosmology, Stanford University, 452 Lomita Mall, Stanford, CA 94305, USA
}

(Received 27 January 2019; Accepted March 15, 2019)

Submitted to ApJ

\begin{abstract}
The Seyfert Galaxy Mrk 335 is known for its frequent changes of flux and spectral shape in the $\mathrm{X}$-ray band occurred during recent years. These variations may be explained by the onset of a wind that previous, non-contemporaneous high-resolution spectroscopy in X-ray and UV bands located at accretion disc scale. A simultaneous new campaign by XMM-Newton and HST caught the source at an historical low flux in the X-ray band. The soft X-ray spectrum is dominated by prominent emission features, and by the effect of a strong ionized absorber with an outflow velocity of 5$6 \times 10^{3} \mathrm{~km} \mathrm{~s}^{-1}$. The broadband spectrum obtained by the EPIC-pn camera reveals the presence of an additional layer of absorption by gas at moderate ionization covering $\sim 80 \%$ of the central source, and tantalizing evidence for absorption in the $\mathrm{Fe} \mathrm{K}$ band outflowing at the same velocity of the soft X-ray absorber. The HST-COS spectra confirm the simultaneous presence of broad absorption troughs in CIV, Ly $\alpha, \mathrm{Ly} \beta$ and OVI, with velocities of the order of $5000 \mathrm{~km} \mathrm{~s}^{-1}$ and covering factors in the range of $20-30 \%$. Comparison of the ionic column densities and of other outflow parameters in the two bands show that the X-ray and UV absorbers are likely originated by the same gas. The
\end{abstract}

Corresponding author: Anna Lia Longinotti annalia@inaoep.mx 
resulting picture from this latest multi-wavelength campaign confirms that Mrk 335 undergoes the effect of a patchy, medium-velocity outflowing gas in a wide range of ionization states that seem to be persistently obscuring the nuclear continuum.

Keywords: Active Galaxies: general — Active Galaxies: Mrk 335 


\section{INTRODUCTION}

Accretion onto supermassive black holes is commonly regarded as one of the most distinctive feature of Active Galaxies (AGN). Nonetheless, the apparently counteractive process of ejection has been gaining growing importance in our knowledge of the AGN phenomenology thanks to the many observational results that have emerged in the last two decades. Ejection of gas in the form of winds is now a common property of radio-quiet AGN and it is particularly prominent in X-ray and UV spectra of local sources (see Crenshaw et al. (2003) for a review). Association of the X-ray winds to absorption lines detected in the ultraviolet band was historically proposed to relate the properties of the gas in the two bands possibly indicating a common origin (Mathur et al. 1995, 1998). However, the origin of the winds is not uniquely determined in all AGN where this phenomenon is observed: at parsec-scale thermal outflows arise from the molecular torus (Krolik \& Kriss 2001), whereas the accretion disc can launch outflows at a radial distance lower than $10^{3}$ gravitational radii via radiative (Proga \& Kallman 2004) and magnetohydrodynamical mechanisms (Königl \& Kartje 1994; Fukumura et al. 2010).

In the X-ray domain, ionization state, velocity and column density of the outflowing gas are relatively easy to measure. They provide information on the physical properties and, partly, on the location of the outflows although the radial distance cannot be measured directly in the spectra unless variability of the ionizing continuum takes place (e.g. Krongold et al. 2007). When the distance of the wind is sufficiently well pinned down, from the velocity and the column density it is possible to estimate the mass and energy output expelled by the outflow and quantify the impact that gas ejection may have onto the host galaxy. This is particularly important to understand the role of AGN winds in feedback process (see King \& Pounds (2015) for a review). The multitude of results obtained via X-ray spectroscopy of bright AGN have shown that "slow" outflows of ionized gas, widely known as "warm absorbers", do not reach the minimum energy output required to alter the AGN star formation activity (e.g. Krongold et al. (2007, 2010)). Instead, the so-called Ultra Fast Outflows (UFO), which are significantly faster and more massive, were shown to be capable of triggering AGN feedback (Tombesi et al. 2015; Feruglio et al. 2015; Longinotti et al. 2018).

In the very recent years, another flavour of AGN winds is being observed and studied: with outflow velocity half-way between warm absorbers and UFOs, obscuring and intermittent outflows produced by clouds orbiting in the Broad Line Region (BLR) are now revealed in the X-ray and UV spectra of Seyfert Galaxies that undergo important flux and spectral variations on relatively short time scales. Mrk 335 (Longinotti et al. 2013), NGC5548 (Kaastra et al. 2014), NGC 985 (Ebrero et al. 2016), NGC3783 (Mehdipour et al. 2017; Kriss et al. 2018) are some examples of obscuration produced by intervening ionized gas in form of "eclipsing" winds.

The close-by Narrow Line Seyfert 1 Galaxy Mrk 335 ( $z=0.025785$, Huchra et al. 1999) is one of the few sources where the emergence of an obscuring wind outflowing at a velocity of 5$6000 \mathrm{~km} \mathrm{~s}^{-1}$ was revealed in the X-ray and UV bands (Longinotti et al. 2013), based on nonsimultaneous observations obtained by XMMNewton and the Hubble Space Telescope. This work also provided the first record of X-ray ionized absorption seen in gratings spectra in Mrk 335, a source that in its past UV and Xray history had showed little evidence for the presence of a typical warm absorber.

Previous works based on CCD-resolution Xray spectra of Mrk 335 were focussed to model 
in great detail the spectral curvature in terms of intervening gas partially covering the line of sight, and/or relativistically blurred reflection from the accretion disk (e.g. Turner et al. 1993; Grupe et al. 2007, 2008; Larsson et al. 2008; Grupe et al. 2012; Gallo et al. 2013, 2015; Wilkins et al. 2015; Gallo et al. 2019).

Indeed, in the past Mrk 335 was mostly known as a typical bright Seyfert 1 Galaxy (e.g. Longinotti et al. 2007; O'Neill et al. 2007) with relativistic Fe K features and negligible intervening absorption until the year 2007 when Swift discovered it in a very low X-ray flux state (Grupe et al. 2007). The ongoing monitoring with Swift since the sudden drop in 2007 has shown that Mrk 335 has remained in this dim X-ray state with some occasional variability detected along the elapsed $\sim 11$ years (Gallo et al. 2018). According to the monitoring reported by these authors, the long dimmed $\mathrm{X}$-ray state has not been accompanied by corresponding variability in the Optical/UV band, which on average stays similar to measurements obtained prior to 2007. On the contrary, repeated X-ray flaring and dipping episodes have triggered several deep follow-up observations with XMM-Newton, Suzaku and NuSTAR (e.g. Parker et al. 2014; Gallo et al. 2015; Wilkins \& Gallo 2015; Keek \& Ballantyne 2016; Komossa et al. 2017, and references above).

In the present paper the outcome of a more recent simultaneous campaign performed on Mrk 335 by XMM-Newton and HST is presented. The chief goal of this campaign was to determine the properties of the absorbers with contemporaneous X-ray and UV data.

The XMM-Newton and HST observations of Mrk 335 were triggered in December 2015 following a decrease of the UV and X-ray flux revealed by Swift (Grupe et al. 2015). XMMNewton started observing on December the $30 \mathrm{~h}$ for a total duration of $\sim 140 \mathrm{ks}$ (OBSID 0741280201) and HST followed on 4 and 7 of

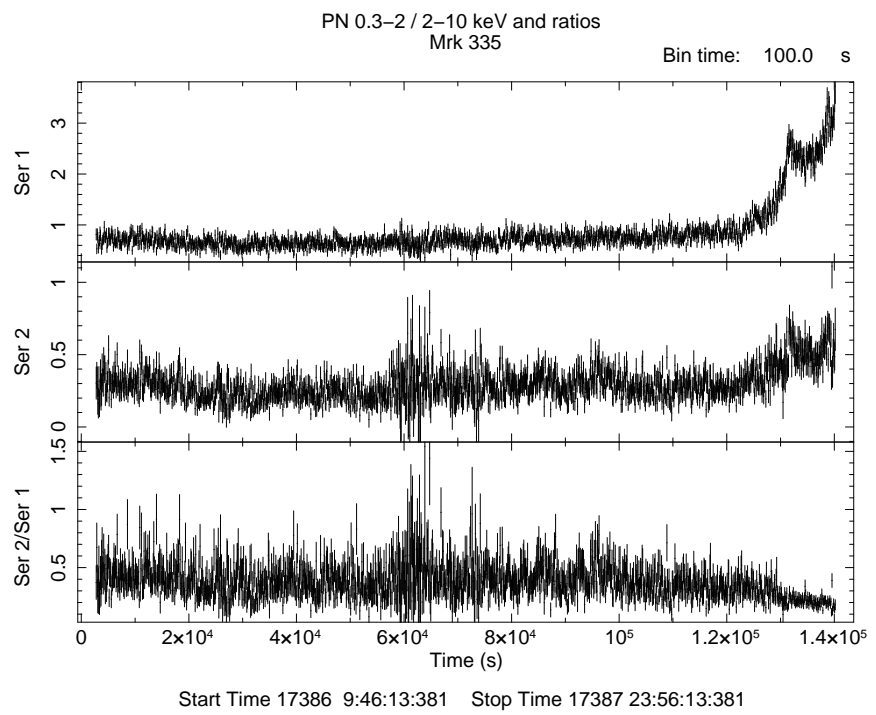

Figure 1. Background-subtracted light curve of Mrk 335 during the XMM-Newton observation. From top to bottom: source counts in $0.3-2 \mathrm{keV}$, $2-10 \mathrm{keV}$ and hardness ratio. The flux rises in the last $20 \mathrm{ks}$ of the observation.

January 2016 for a total of 7 orbits. Unlike the previous report (Longinotti et al. 2013) that relied on archival data obtained 4 months apart, the above timing provides quasi-simultaneity between the observational properties in UV and X-ray bands.

\section{XMM-NEWTON OBSERVATION AND SPECTRAL ANALYSIS}

The XMM-Newton EPIC cameras were both set to operate in Full Window mode. Data were processed with SAS 16.0.0 $0^{1}$. Both pn and MOS detectors were exposed to strong protons flares during some portions of the light curve. We followed the procedure recommended in the Science Threads to clean the raw event file and we obtained a clean exposure of $115 \mathrm{ks}$ in the pn camera. Data obtained by the RGS instrument were not affected by the flaring periods. Spectra were produced by standard SAS tools (rgsproc, epproc, emproc). EPIC source and background counts were extracted

\footnotetext{
${ }^{1}$ https://www. cosmos.esa.int/web/xmm-newton/sas
} 


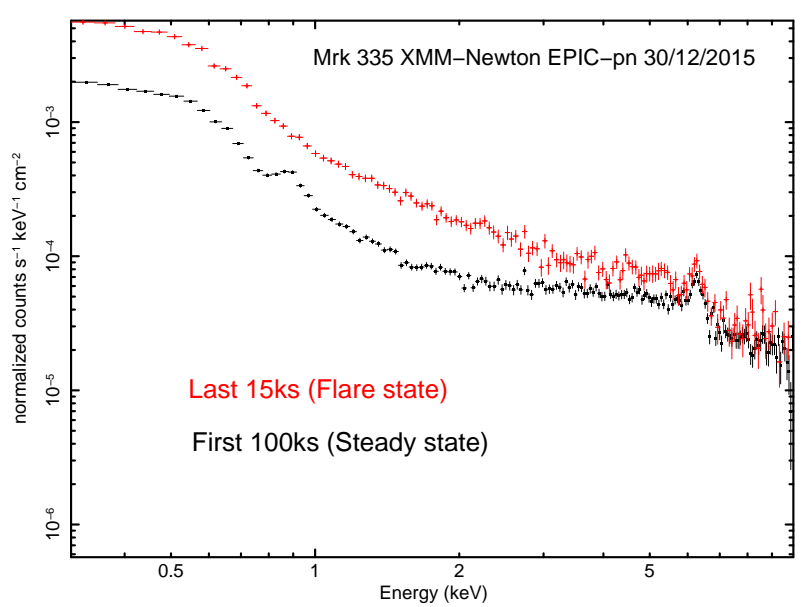

Figure 2. EPIC pn spectra extracted from the steady part of the light curve (first $\sim 100 \mathrm{ks}$ of the observation) and from the "flare" (last $15 \mathrm{ks}$ ). The Fe $\mathrm{K}$ band is moderately affected by the spectral change induced by the rise of flux. Both spectra are severely affected by high background above $8 \mathrm{keV}$.

from regions of 40 arcsec of radius, including all patterns (0-4 for the pn and 0-12 for the MOS cameras). After checking consistency among EPIC spectral products, the analysis of MOS spectra was not included in this paper.

The light curve extracted from the pn counts shows an increase of the flux of a factor of $\sim 3$ that happened during the last $\sim 20 \mathrm{ks}$ of the observation (Fig. 1) We therefore split the data into "steady" (first $~ 120 \mathrm{ks}$ ) and "flare" (last $\sim 20 \mathrm{ks}$ ) states to check for possible spectral variations. The spectral analysis described in the following was applied consistently to both flux states and model parameters were compared. Due to the combined effect of the source being at a very low flux state and of the short exposure in the spectral products extracted from the flaring portion of the light curve flare (20 ks for the RGS and $15 \mathrm{ks}$ for the pn), no significant variation was detected in the spectral parameters corresponding to the two flux states.

However, as extensively described in the following section, the spectrum extracted from the "steady" interval of the light curve is completely dominated by emission lines (see Fig. 3 and Ta- ble 1) that are not entirely recovered in the spectrum extracted from the "flare" because the continuum emission starts covering them as the flux rises. This effect can be visualized in Fig. 2 . With the aim of obtaining an accurate characterization of the soft X-ray features, the spectral analysis was applied to the "steady" interval ( $120 \mathrm{ks}$ for the RGS and $\sim 100 \mathrm{ks}$ for the pn). After obtaining a sound characterization of the underlying soft X-ray features a general consistency of this spectrum with the the "flare" interval was confirmed. Since the inclusion of the "flare" interval does not provide any tighter constraints on our spectral analysis, we decided not to include it in the present analysis and to focus on the results obtained on the "steady" interval, which represent the most genuine description of the lowest X-ray spectrum of Mrk 335 so far obtained. The detailed analysis of the "flare" interval is reported by Gallo et al. (2019).

The spectral analysis was carried out with XSPEC version 12.9.1 (Arnaud 1996).

The analysis of the grating spectrum was carried out on the combined RGS1+RGS2 data sets obtained through the SAS tool rgscombine. Counts were not binned, therefore the Cash Statistics was applied (Cash 1979). The EPIC-pn spectrum was binned by the SAS tool specgroup therefore $\chi^{2}$ statistics was applied throughout its analysis (see Section 2.2) Error bars of spectral parameters are quoted to $1 \sigma$. Fluxes in the $0.3-10 \mathrm{keV}$ and in the $0.3-2 \mathrm{keV}$ bands during the steady part of the XMM-Newton observation were measured to be $\sim 3.7 \times 10^{-12}$ and $\sim 1.1 \times 10^{-12} \mathrm{erg} \mathrm{cm}^{-2}$ $\mathrm{s}^{-1}$ respectively. These fluxes translate to $\mathrm{X}$ ray luminosity of $\mathrm{L}_{0.3-10}=5.5 \times 10^{42} \mathrm{erg} \mathrm{s}^{-1}$ and $\mathrm{L}_{0.3-2}=1.5 \times 10^{42} \mathrm{erg} \mathrm{s}^{-1}$.

\subsection{The high resolution X-ray spectrum}

The entire RGS spectrum is plotted in Figure 3. The spectrum is dominated by very intense emission lines and Radiative Recombination Continua (RRC) features, along with 


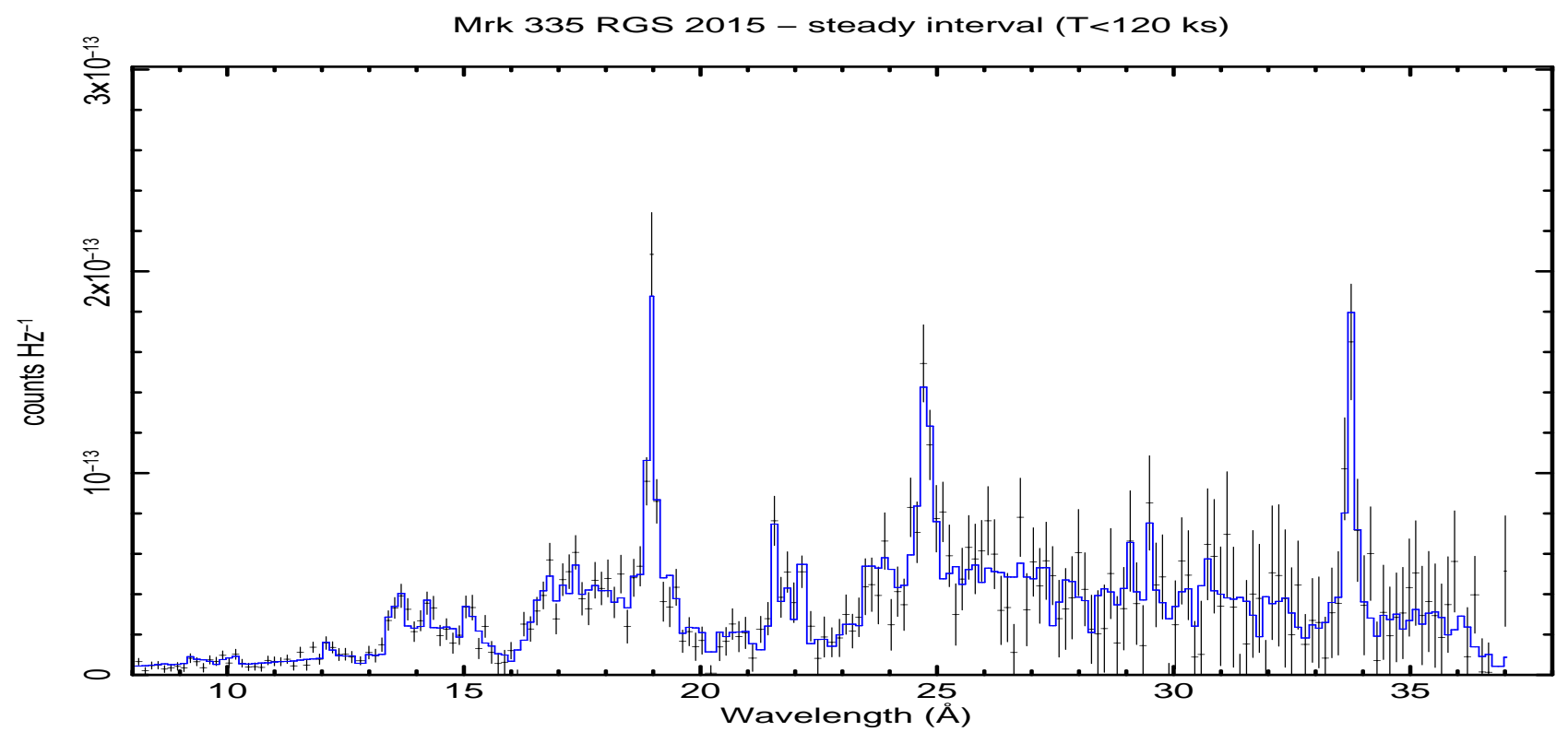

Figure 3. Best fit of the RGS spectrum corresponding to the steady interval of the light curve. The continuum is modeled with a power law with $\Gamma=2.72$, the model includes the emission features and the ionized absorber described in Section 2.1. Data have been binned for plotting purpose.

strong signature of ionized absorption. The emission component was discovered in a previous low-flux, shorter observation by XMMNewton in 2007 (Grupe et al. 2008) and reported by Longinotti et al. (2008). The higher signal-to-noise of the present data allows the effect of absorption lines to be clearly distinguished in the continuum emission. This is not surprising since in another previous XMMNewton observation obtained in 2009 the emergence of a strong multi-layer ionized absorber was revealed in both X-rays and UV bands (Longinotti et al. 2013).

The latest (2015) RGS view of Mrk 335 shows clear signatures of both features, therefore we constructed a model that includes emission and absorption guided by our previous knowledge of the spectrum.

To fit the soft X-ray continuum in the range 7-38 A we start by including a power law with $\Gamma=2.8$ that is absorbed by a Galactic column density fixed to $3.6 \times 10^{20} \mathrm{~cm}^{-2}$ (Kalberla et al. 2005). The Galactic absorption is modeled by the TBabs component included in the suite of ISM absorption model developed by Wilms et al. (2000).

\subsubsection{The emission features}

To this continuum, several narrow Gaussian lines are added to fit the emission lines. We initially included all the atomic transitions reported by Kinkhabwala et al. (2002) for the Seyfert 2 Galaxy NGC 1068. Many of them are detected in Mrk 335 spectrum and the resulting list of emission lines with significance $\Delta \mathrm{C}_{\text {stat }}>1$ (one free parameter) is reported in Table 1. Line widths were initially set to $1 \mathrm{eV}$ and line positions were fixed to their respective rest wavelength, therefore only the flux of the line is left as a free parameter. A close inspection of the Ly $\alpha$ transitions from $\mathrm{H}$-like ions lines in Fig. 4 indicates that an underlying broader component is needed. Indeed, when the width of these lines is left free, all of them are best described by a broad component with Full Width Hald Maximum (FWHM) of around $2000 \mathrm{~km} \mathrm{~s}^{-1}$. We also included Radiative Recombination Continua (RRC) features by adding 4 redge components at the expected 

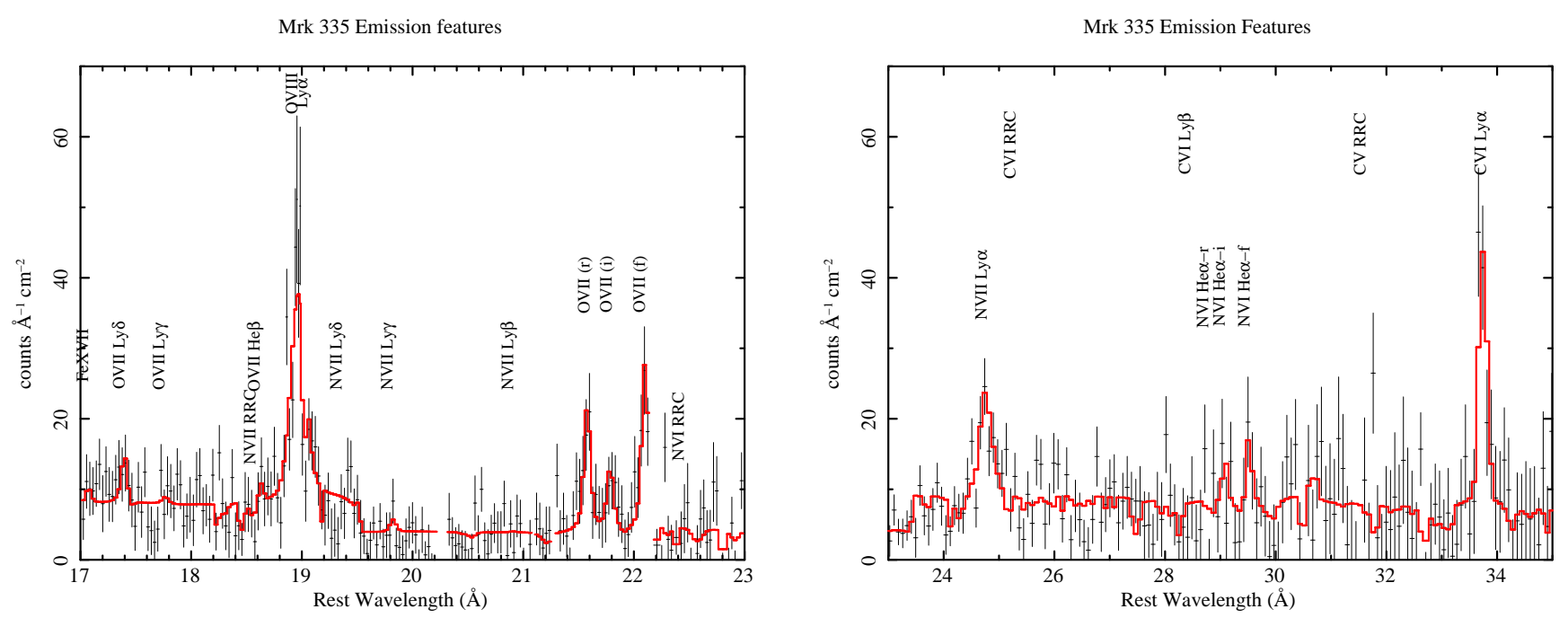

Figure 4. Close up on the softest region of RGS spectrum. The labels mark only emission features, the warm absorber is included in the model but labels have been omitted to avoid overlapping with emission lines markers.

position of the CV, CVI, OVII and OVIII RRCs (see Table 2).

This model including only the emission component and the power law continuum yields $\mathrm{C}_{\text {stat }} /$ d.o.f. $=3353 / 2998$ and it is adopted in the following as a mere phenomenological description of the spectral features in emission. A close up on the emission component is plotted in Fig. 4. Further detailed analysis of the properties of the emission features is deferred to a forthcoming publication.

\subsubsection{The warm absorber}

The effect of line-of-sight absorption was then modeled by employing the suite of photoionization models PHASE (Krongold et al. 2003) and after constructing the spectral energy distribution of the source that is necessary to calculate the ionization balance assumed to compute the absorption spectrum. From Longinotti et al. (2013) we are well aware of the presence of a complex multi-component warm absorber in this source. In order to allow a straightforward comparison of the present warm absorber properties with past epochs, we adopted the same spectral energy distribution described by Longinotti et al. (2013). The UV-X-ray SED was built assuming the simultaneous fluxes and
Table 1. Emission lines detected in RGS spectrum.

\begin{tabular}{cccc}
\hline \hline ID & Rest & Flux & $\Delta$ Cstat \\
- & Wavelength & $\left(10^{-5} \mathrm{ph} \mathrm{cm}^{-2} \mathrm{~s}^{-1}\right)$ & - \\
\hline Mg XI He $\alpha$ & 9.228 & $0.247_{-0.224}^{+0.245}$ & 3.23 \\
Ne X Ly $\alpha$ & 12.134 & $0.555_{-0.270}^{+0.298}$ & 13.12 \\
NeIX He $\alpha r$ & 13.447 & $0.420_{-0.211}^{+0.228}$ & 11.37 \\
NeIX $i$ & 13.553 & $0.120_{-0.120}^{+0.198}$ & 1.12 \\
NeIX $f$ & 13.698 & $0.391_{-0.210}^{+0.226}$ & 10.37 \\
Fe XVII & 15.015 & $0.301_{-0.193}^{+0.213}$ & 6.60 \\
Fe XVII & 17.073 & $0.225_{-0.224}^{+0.242}$ & 2.73 \\
OVII He $\delta$ & 17.396 & $0.462_{-0.252}^{+0.275}$ & 9.57 \\
OVII He $\gamma$ & 17.768 & $0.207_{-0.207}^{+0.259}$ & 1.93 \\
OVII He $\beta$ & 18.627 & $0.215_{-0.215}^{+0.266}$ & 1.97 \\
OVIII Ly $\alpha$ & 18.969 & $3.467_{-0.515}^{+0.536}$ & 146.87 \\
NVII Ly $\gamma$ & 19.826 & $0.346_{-0.346}^{+0.418}$ & 2.37 \\
OVII He $\alpha r$ & 21.580 & $2.325_{-0.762}^{+0.833}$ & 28.97 \\
OVII $i$ & 21.790 & $1.250_{-0.619}^{+0.693}$ & 12.04 \\
OVII $f$ & 22.101 & $3.227_{-0.873}^{+0.979}$ & 50.15 \\
NVII Ly $\alpha$ & 24.781 & $2.270_{-0.992}^{+0.905}$ & 17.25 \\
NVI He $\alpha i$ & 29.083 & $0.609_{-0.609}^{+0.904}$ & 1.35 \\
NVI $f$ & 29.534 & $1.163_{-0.840}^{+0.945}$ & 4.93 \\
CVI Ly $\alpha$ & 33.736 & $7.314_{-1.894}^{+2.039}$ & 45.85 \\
\hline \hline
\end{tabular}

spectral shape from the Optical Monitor photometry and the EPIC-pn spectrum of 2009 shown in Fig. 4 of Longinotti et al. (2013). This choice is also supported by the long term behav- 
Table 2. RRC in the RGS spectrum

\begin{tabular}{ccc}
\hline \hline ID & Flux & $\Delta$ Cstat \\
- & $\left(\mathrm{ph} \mathrm{cm}^{-2} \mathrm{~s}^{-1}\right)$ & - \\
\hline $\mathrm{CV}$ & $<3.21 \times 10^{-5}$ & 1.4 \\
$\mathrm{CVI}$ & $<1.48 \times 10^{-5}$ & 4.5 \\
OVII & $9.35_{-3.37}^{+3.58} \times 10^{-6}$ & 22 \\
OVIII & $3.44_{-2.14}^{+2.30} \times 10^{-6}$ & 9 \\
\hline \hline
\end{tabular}

ior of Mrk 335 reported by Gallo et al. (2018), which is summarized in Section 1.

The absorber in PHASE is described by the following parameters: the gas ionization state defined as $\mathrm{U}=\frac{Q}{\left(4 \pi R^{2} c n_{e}\right)}$ (with $\mathrm{Q}$ as the ionizing luminosity, $\mathrm{n}_{e}$ as the gas electron density and $\mathrm{R}$ as the distance of the outflowing gas from the X-ray source), the column density $\mathrm{N}_{H}$, the turbulent velocity $\mathrm{v}_{\text {broad }}$ and the outflow velocity. Indeed, the addition of an ionized absorber with initial best fit parameter $\log \mathrm{U} \sim 0.8$ and $\log \mathrm{N}_{H} \sim 21.8$ produces an improvement of $\Delta \mathrm{C}_{\text {stat }}=66$ when compared to the model including the power law continuum and the emission component.

The detailed warm absorber parameters and errors are reported in Table 3. The outflow velocity of this absorber is of the order of 5$6 \times 10^{3} \mathrm{~km} \mathrm{~s}^{-1}$, consistent with the velocity measured in the 2009 spectra by Longinotti et al. (2013). The velocity broadening of the lines in the absorber was initially set to $100 \mathrm{~km} \mathrm{~s}^{-1}$ and then left free to vary. A moderately tight constraint on this parameter could be found: $\mathrm{v}_{\text {broad }} \leq 170 \mathrm{~km} \mathrm{~s}^{-1}$, therefore in the rest of the analysis this parameter is kept fixed to $100 \mathrm{~km} \mathrm{~s}^{-1}$. The slope of the underlying power law continuum is $2.72 \pm 0.18$. Spectra plotted in Figure 3 and 4 include the effect of this layer of absorption. The final fit statistic for the model including the power law continuum, the ionized absorber and the emission component is $\mathrm{C}_{\text {stat }} /$ d.o.f. $=3287 / 2992$.
Fig. 5 displays the most intense absorption features that are driving the warm absorber. The strongest absorption feature (left panel) is due to a blend of several lines resulting from Mshell transitions in mildly ionized FeI-XVI, the so-called Fe UTA (Unresolved Transition Array Netzer 2004). Further absorption is imprinted by transitions of CVI, NVII, OVII, OVIII. The question mark labels plotted in the left panel of Fig. 5 mark the position of two unidentified absorption lines that could not be fitted self-consistently with another absorption component despite several attempts of finding a coherent model for these features. They will not be discussed in the remainder of this paper.

An exhaustive comparison of the (several) multi-epoch X-ray data sets of Mrk 335 is out of the present scope and it will be the subject of a forthcoming publication. However, in Section 4 we will review the properties of the present warm absorber (year 2015) compared to the findings reported by Longinotti et al. (2013) on the absorber emerged in Mrk 335 in the year 2009.

Table 3. Best fit parameters of the RGS warm absorber.

\begin{tabular}{cccc}
\hline \hline $\log \mathrm{U}^{(a)}$ & $\begin{array}{c}\log \mathrm{N}_{H} \\
\left(\mathrm{~cm}^{-2}\right)\end{array}$ & $\begin{array}{c}\mathrm{v}_{\text {out }} \\
\left(\mathrm{km} \mathrm{s}^{-1}\right)\end{array}$ & $\begin{array}{c}\text { v broad } \\
\left(\mathrm{km} \mathrm{s}^{-1}\right)\end{array}$ \\
\hline $0.85_{-0.14}^{+0.09}$ & $21.82_{-0.19}^{+0.20}$ & $5700_{-400}^{+800}$ & $100(\mathrm{f})$ \\
\hline \hline
\end{tabular}

\subsection{The broadband $X$-ray spectrum}

The bandpass of the RGS instrument is limited to below $2.5 \mathrm{keV}$, therefore to achieve a full understanding of the entire spectrum we applied the RGS best fit model to the EPIC-pn data. This data is shown in Fig.2. The RGS model offers a very detailed description of the ionized gas that is responsible for emission and absorption in the soft X-ray band. Nonetheless the extension of the bandwidth up to $10 \mathrm{keV}$ 

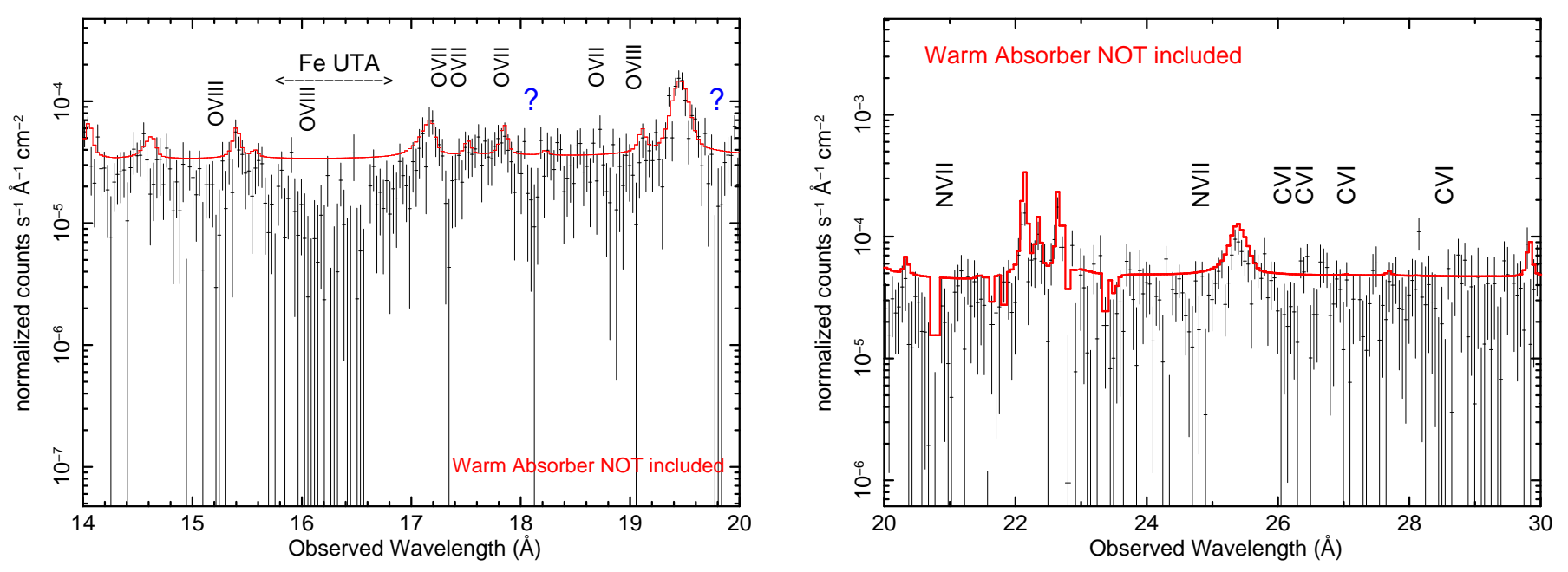

Figure 5. Region of the RGS spectrum mostly affected by the warm absorber: the spectrum is fitted only by the continuum plus the emission component and labels mark the most prominent absorption features. The corresponding emission lines are displayed in Fig. 4. Question marks highlight the positions of unidentified absorption lines. Data are binned for plotting purpose.

immediately reveals the effect of unseen spectral components that are missing in this initial model due to bandpass limitation.

We start by adding to the RGS model a Gaussian emission line to accommodate strong residuals corresponding to a $\mathrm{Fe} \mathrm{I} \mathrm{K} \alpha$ line that is highly prominent in this source (see details in Section 2.2.2). We then added a blackbody component (bbody) to fit -at least phenomenologically- the strong soft excess that has always been present in X-ray data of Mrk 335 (e.g. Bianchi et al. 2001; Grupe et al. 2008; Gallo et al. 2015; Chainakun \& Young 2015). This addition has the effect of flattening the underlying power law that is now extended to fit the hard X-ray band and that presents a photon index of $\Gamma \sim 0.9$. The parameters of the blackbody component are in the range of the standard values for Seyfert 1 sources: the temperature is $\mathrm{kT}=0.11 \pm 0.02 \mathrm{keV}$. We note that the use of the more realistic Comptonized blackbody model (comptt) does not produce significant changes in the spectral fit, therefore we kept the more basic parametrization with bbody to account for the soft excess. Finally, guided by residuals around $1.7-2 \mathrm{keV}$, we added 4 Gaussian emission lines at the positions of

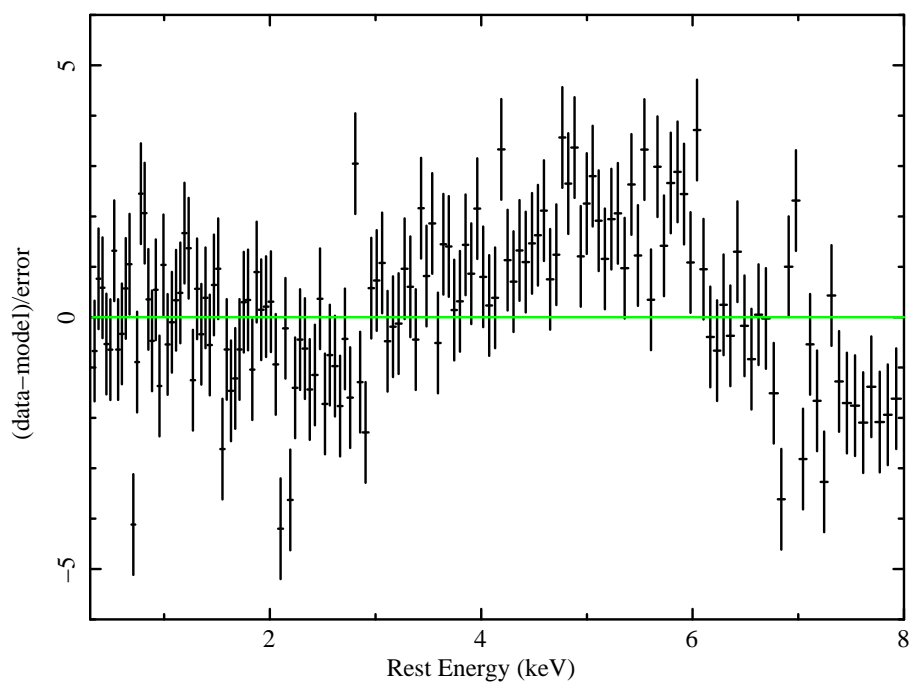

Figure 6. Residuals of the pn spectrum obtained before including the partial covering and the reflection component in the continuum model (see 2.2.

$6.182,6.740,7.130,8.421 \AA$ to accommodate the transitions of SiXIV Ly $\alpha$, SiXIII He $\alpha f$, Si I K $\alpha$ and MgXII Ly $\alpha$. These emission lines cannot be detected in the grating spectrum as they fall out of the bandpass. Nonetheless, considering the realm of features in Tables 1 and 2 along with examples of other AGNs where the emission spectrum could be measured in a wider band (e.g. NGC 4151 Ogle et al. 2000), the presence of emission from heavier elements 
in the pn data of Mrk 335 is highly likely and indeed, their inclusion significantly improves the residuals in this spectral region.

Even with these modifications, both the fit statistics of $\chi^{2} /$ d.o.f. $=416 / 141$ and the hard Xray curvature in the residuals (see Fig.6) suggest the presence of additional continuum component(s).

\subsubsection{Partial covering absorber and Reflection component}

To mimic the effect of mildly ionized gas partially covering the primary X-ray continuum we applied to the power law an additional layer of absorption parametrized by a second PHASE component with an initial low ionization parameter and a variable covering factor. This partial covering component significantly improves the spectral fit $\left(\Delta \chi^{2}=220\right.$ for 3 d.o.f.) and the intrinsic power law gets to a steeper photon index more typical of Mrk 335, $\Gamma=1.65 \pm 0.11$. The column density of this gas is found to be quite high, $\log \left(\mathrm{N}_{H}\right)=22.99 \pm 0.06$, and the covering factor is $0.79_{-0.05}^{+0.02}$. The ionization parameter could not be constrained precisely (see Fig. 7) but the upper limit of $\log \mathrm{U}$ points to a degree of ionization lower than $\log \mathrm{U} \sim 1.35$. Likewise, the velocity of this absorber could not be measured due to the limited resolution of the pn CCD, therefore we kept it fixed to the same value of the RGS warm absorber $\left(\sim 5600 \mathrm{~km} \mathrm{~s}^{-1}\right)$. We note that testing alternative velocities (e.g. $\mathrm{v}_{\text {out }}=2000$ or 800 $\mathrm{km} \mathrm{s}^{-1}$ ) does not provide any relevant change in the spectral fit.

During the fitting process, some parameters of the ionized absorber detected in the RGS have been frozen: the ionization parameter, outflow velocity and velocity broadening are frozen to the best fit values reported in Table 3, while the column density is left free. This is justified by considering that the coarser resolution of the CCD cannot improve the parameters already well constrained by grating spectroscopy.

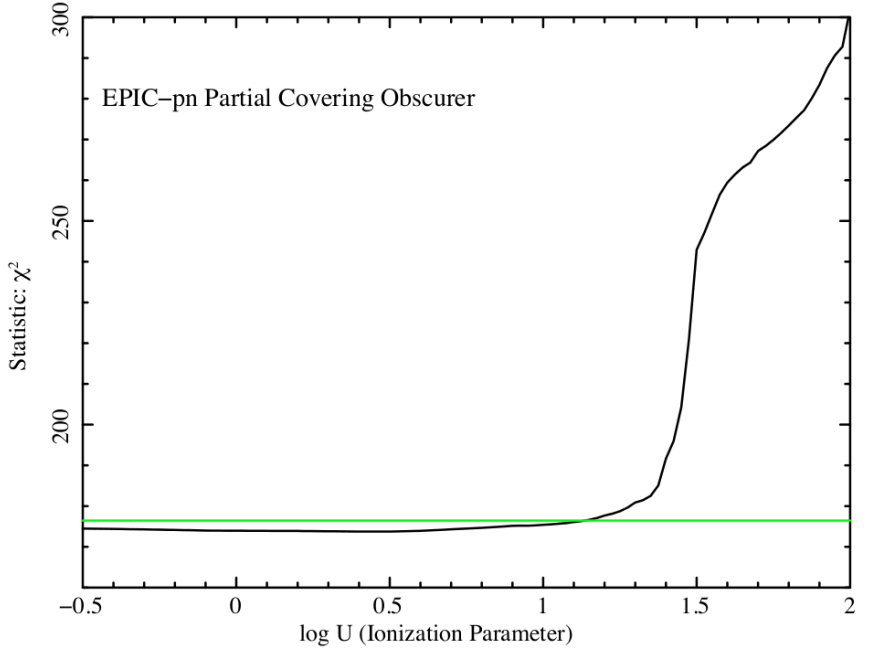

Figure 7. Ionization parameter of the EPIC-pn partial covering absorber plotted against fit statistics of the best fit model $\left(\chi^{2} /\right.$ d.o.f. $\left.=174 / 138\right)$ that includes the reflection component (last paragraph of Section2.2.

As a conclusive and necessary step of the analysis of the broadband continuum, we also considered the presence of a Compton reflection component. A detailed analysis of the reflection spectrum and the property of the inner accretion disc of Mrk 335 is out of the scope of this paper and it is presented elsewhere (Gallo et al. 2019). However, the presence of inner relativistic reflection in Mrk 335 was intensively studied in recent years (Kara et al. 2013; Parker et al. 2014; Gallo et al. 2015) and eventually confirmed as one of the dominant spectral component of this AGN. Therefore we included a basic parameterization of the reflection spectrum by removing the partial covering and by adding a pexrav component to the broadband model. This test yields a reduced $\chi^{2}$ of 3.38 and a much flatter power law $(\Gamma \sim 1.26)$, indicating that partial obscuration is still required by the data. Once the partial covering is included back into the model with the reflection component, the slope of the continuum goes to $\Gamma=2.14_{-0.13}^{+0.10}$ and the fit statistics 
improves to $\chi^{2} /$ d.o.f. $=174 / 138$. The broadband model is plotted in Figure 8. We remark that this parametrization serves merely to test the statistical requirement of the partially covering gas, therefore a detailed spectral fitting is not envisaged herein and standard reflection parameters are adopted: the cut-off energy is $500 \mathrm{keV}$, solar abundances are chosen for the elements heavier than $\mathrm{He}$ and for $\mathrm{Fe}$, and the inclination angle of the disk is fixed to 30 degrees. The only fitting parameter left free is the reflection fraction that, not surprisingly, pegs to its maximum value $(R=10)$ indicating a dominant contribution from the inner accretion disk. These values are broadly consistent with what reported by Parker et al. (2014), who, in their relativistic treatment, had found high reflection fractions in NuSTAR data of Mrk 335 at very low flux state. This behavior is also reported by the recent publication by Gallo et al. (2019), to which the reader is deferred for a more detailed analysis of the reflection properties of the source. Finally, we note that our coarse parametrization does not exclude the likely contribution of a more distant reflector from the outer part of the disk or the molecular torus of the AGN, as indicated by the narrow $\mathrm{Fe} \mathrm{K} \alpha$ line reported in next section.

\subsubsection{The Iron line band}

We now take a closer look at the Fe $\mathrm{K}$ band. Owing to the spectral complexity of the soft Xray band and with the aim to speed up the fitting procedure, the following analysis was carried out on the data within the range $3-8 \mathrm{keV}$. This is justified by considering that the opacity of the soft X-ray warm absorber has no effect above $3 \mathrm{keV}$ and that the bulk of strong emission lines are emitted below this energy threshold. The continuum model from the previous section constituted by a partially covered power law plus Compton reflection has been applied to this restricted band (the blackbody component was dropped since it has no effect in this band).

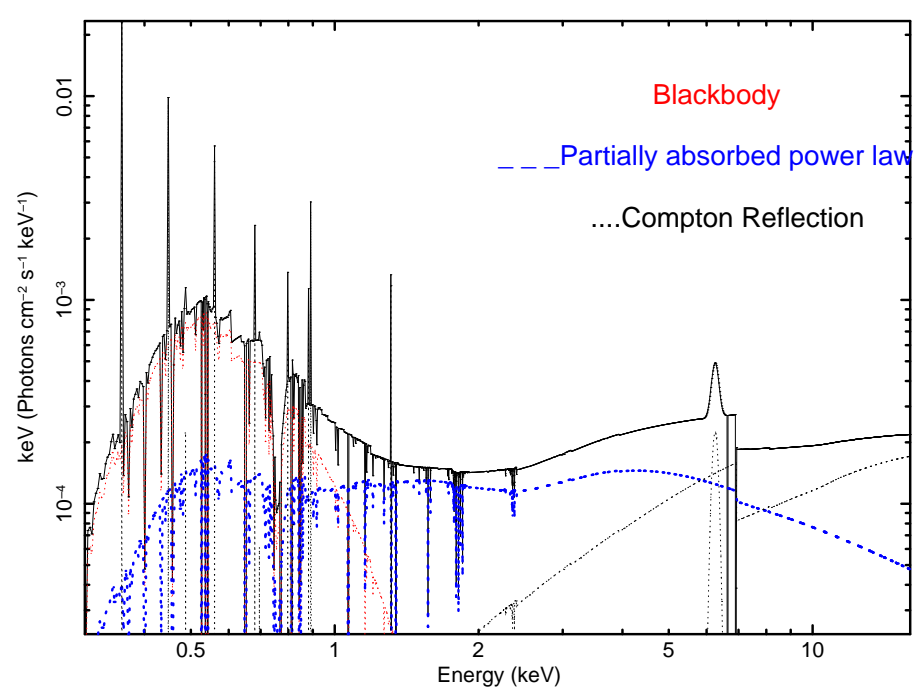

Figure 8. Broadband model applied to the EPICpn spectrum. Solid black: combination of all spectral components. Red: Blackbody + warm absorber detected in RGS. Blue: power law absorbed by mildly ionized gas covering $\sim 80 \%$ of the $\mathrm{X}$ ray source and by warm absorber. Dotted black: Compton Reflection. Soft X-ray emission features are modeled by Gaussian lines. For simplicity, the $\mathrm{Fe} \mathrm{K}$ absorption is also modeled by two Gaussian lines.

The choice of not extending the bandwidth to the nominal $10 \mathrm{keV}$ is due to the rising of instrumental background above $8 \mathrm{keV}$ that introduces significant uncertainty in the spectral features apparently present above this threshold.

The presence of the $\mathrm{K} \alpha$ emission line from neutral Iron is very evident in the spectrum and it has been fitted with a Gaussian profile with peak energy $\mathrm{E}=6.41_{-0.02}^{+0.03} \mathrm{keV}$ and width $\sigma=0.12 \pm 0.03 \mathrm{keV}$. The intensity of the $\mathrm{Fe} \mathrm{K} \alpha$ line parametrized with this Gaussian profile and expressed as its Equivalent Width is $\mathrm{EW}=300 \pm 45 \mathrm{eV}$. The continuum model after the inclusion of the $\mathrm{Fe} \mathrm{K} \alpha$ line yields a fit statistics of $\chi^{2} /$ d.o.f. $=97 / 74$. The spectrum fitted by this model is plotted in Fig. 9. The Fe line parameters are compatible with emission in the molecular torus via Compton reflection, as proposed by O'Neill et al. (2007) for the high flux state of Mrk 335. We note that the con- 
tribution of a distant reflector was not directly tested via spectral fitting in the present data but it is discussed in Gallo et al. (2019).

Additional residuals on the blue side of the Fe $\mathrm{K} \alpha$ line suggest us to explore the presence of emission from highly ionized Iron, which was already revealed when the source was observed in high flux state with higher photon statistics (O'Neill et al. 2007). We added a narrow $(\sigma=1$ eV) Gaussian line in emission and measured its position at $\mathrm{E}=6.96_{-0.16}^{+0.05} \mathrm{keV}$, but only an upper limit of $\mathrm{EW} \leq 10 \mathrm{eV}$ could be measured, therefore this line is no longer included in the following tests.

We then proceed to examine the residuals in absorption that are still present in the spectrum. Indeed the addition of a narrow $(\sigma=1 \mathrm{eV})$ Gaussian line with negative intensity at a redshift-corrected position of $\mathrm{E}=7.15 \pm 0.09 \mathrm{keV}$ ("abs1" in Fig. 9) improves the fit statistics by $\Delta \chi^{2}=8$ (for 2 d.o.f.) and its intensity is measured to $\mathrm{EW}=57 \pm 30 \mathrm{eV}$. A second absorption line is found at the position of $\mathrm{E}=6.82 \pm 0.05 \mathrm{keV}$ ("abs2" in Fig. 9) with an intensity of $\mathrm{EW}=68 \pm 25 \mathrm{eV}$ and statistical improvement of $\Delta \chi^{2}=10$ for 2 d.o.f.

These absorption features suggest the presence of an ionized blue-shifted absorber that could constitute a high ionization layer of the outflowing system detected in the soft X-ray. The closest transitions that could originate the FeK absorption feature at $7.15 \mathrm{keV}$ are Fe XXVI $\left(\mathrm{E}_{l a b}=6.97 \mathrm{keV}\right)$ and Fe XXV $\left(\mathrm{E}_{l a b}=6.67 \mathrm{keV}\right)$. The corresponding outflow velocity would be respectively $\sim 7,500$ and $20,000 \mathrm{~km} \mathrm{~s}^{-1}$. With regard to the second absorption line at $6.82 \mathrm{keV}$, if interpreted as blue-shifted FeXXV, the outflow velocity would be around $\sim 7000 \mathrm{~km} \mathrm{~s}^{-1}$. Considering these numbers and the large uncertainties in the position of both absorption lines measured in the pn CCD data, the most viable interpretation is that both absorption lines in the Fe $\mathrm{K}$ band correspond to $\mathrm{He}$ and $\mathrm{H}$-like Iron
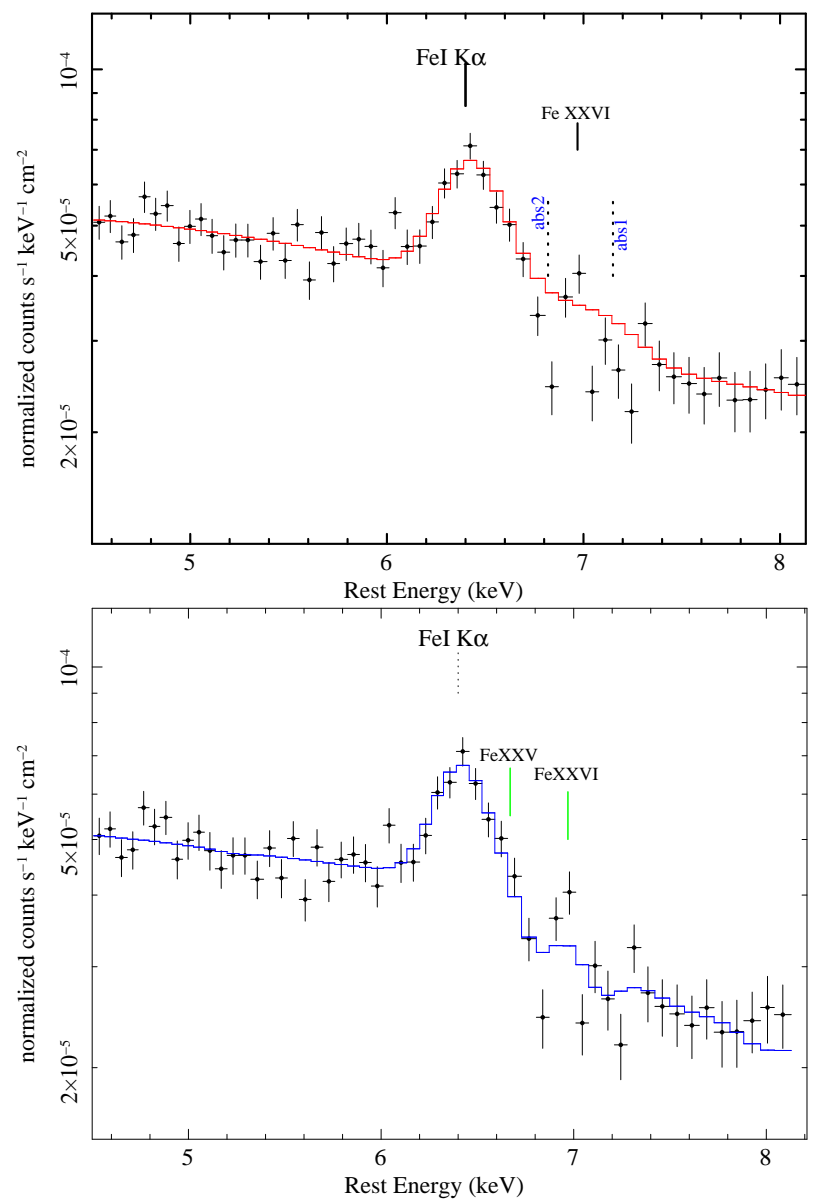

Figure 9. Close up of the Fe $\mathrm{K}$ band in the EPIC pn spectrum. Top: the underlying continuum is modeled as a partially covered power law and only one Gaussian emission line is included to model the Fe I K $\alpha$ line. The laboratory position of the FeXXVI transition is marked by the solid line in black color (see 2.2.2) Dotted vertical lines mark the position of the two absorption lines (blue vertical labels). Bottom: the spectrum is modeled as above but now including the PHASE component of the high ionization absorber with outflow velocity of $5,200_{-200}^{+700} \mathrm{~km} \mathrm{~s}^{-1}$ described at the end of 2.2.2. Green labels mark the laboratory positions of the transitions of highly ionized Fe.

originated in a gas outflowing at a velocity of $\sim$ $7000 \mathrm{~km} \mathrm{~s}^{-1}$, in reasonable agreement with the velocity pattern $\left(5700 \mathrm{~km} \mathrm{~s}^{-1}\right)$ of the warm absorber detected in the grating spectrum.

As a final step, we have replaced the two absorption lines in the spectral model with a PHASE component that can self-consistently fit 
both features. The improvement in the fit statistics corresponds to $\Delta \chi^{2}=19$ for 3 d.o.f., and the parameters of the photoionized wind are $\log \mathrm{U}=3.13_{-0.59}^{+0.09}, \log \mathrm{N}_{H} \geq 23.07$, with an outflow velocity of $5,200_{-200}^{+700} \mathrm{~km} \mathrm{~s}^{-1}$. This velocity, which is now measured self-consistently, shows a much finer agreement with the value derived from the grating spectrum for the low ionization component of the absorber.

\subsubsection{On the consistency of the broadband model with the grating spectrum}

Due to bandwidth restriction, neither of the two EPIC-pn absorbers (the highly ionized and the partial covering) seem to imprint obvious features in the RGS data.

With regard to the partial covering, the bulk of absorption comes from continuum opacity and its effect starts to be visible in the spectrum above $3 \mathrm{keV}$, therefore virtually impossible to be detected in RGS. The inclusion of a PHASE component in the RGS best fit model with parameters fixed to the EPIC-pn values is formally consistent with the data, although no improvement in the fit statistics is found. When the column density and the velocity are kept frozen, $\log \mathrm{U}$ is found around 1.04 with an extremely low covering factor $\left(\mathrm{C}_{f}=0.1\right)$, that would not allow any individual absorption line to be strong enough to be detected in RGS.

We now explore the possible presence of the EPIC-pn highly ionized absorber in RGS data. The inclusion of this component into the RGS best fit model yields a modest improvement of $\Delta \chi^{2}=5$ for 3 free parameters: $\log \mathrm{U}=2.61_{-0.33}^{+0.75}$, $\log \mathrm{N}_{H}=23.4_{-0.99}^{+0.39}$ and $\mathrm{v}_{\text {out }}=6500_{-700}^{+400} \mathrm{~km} \mathrm{~s}^{-1}$. These parameters are broadly consistent with the EPIC-pn values reported at the end of the previous section.

We fully acknowledge that the presence of the high ionization absorber is not statistically robust in any of the spectral data. Nonetheless, after running these checks, we conclude that the simultaneous (albeit moderate) significance in
CCD and grating spectra of an outflow with velocity consistent with the well characterized system of winds observed in RGS, concurs to indicate that a high ionization component of the wind is present in the low flux state of Mrk 335.

\section{HUBBLE SPACE TELESCOPE ULTRAVIOLET SPECTRA}

\subsection{Observations and Data Reduction}

The triggered XMM-Newton observations of Mrk 335 were coordinated with two HST observations. The first of these, on 2016-01-04, followed the XMM-Newton observation by five days. This visit used all COS FUV gratings (G130M, G160M, and G140L) to cover the full wavelength range from $912 \AA$ to $2000 \AA$, specifically including the region surrounding $\operatorname{Ly} \beta$ and O VI. The second visit, another three days later, on 2016-01-07, supplemented the G140L exposures to obtain better $\mathrm{S} / \mathrm{N}$ in the Ly $\beta$ and $\mathrm{O}$ VI region. Table 4 gives the observational details of the individual spectra. Green et al. (2012) describe the key characteristics of the design and performance of the COS instrument on the Hubble Space Telescope (HST). The G130M and G160M gratings have a resolving power of $R \sim 15000$ over the wavelength range of 1135$1800 \AA$. The G140L grating has resolving power $R \sim 2000$ covering $912 \AA$ to $2000 \AA$ with the 1280 central wavelength setting, but with a gap between detector segments from 1190-1265 $\AA$. We chose two central wavelength settings for G130M and G160M to bridge the gap between the FUV detector segments. These settings were also chosen to avoid placing the gap on spectral features of interest in Mrk 335. In addition to multiple central wavelength settings, we also used multiple focal-plane positions to avoid flat-field features and other detector artifacts.

We processed the observations using v3.1 of CALCOS, the COS calibration pipeline, supplemented by custom flat-field files developed for 
Table 4. COS Observations of Mrk 335

\begin{tabular}{lcccc}
\hline \hline Data Set & Grating/Tilt & Date & $\begin{array}{c}\text { Start Time } \\
\text { (GMT) }\end{array}$ & $\begin{array}{c}\text { Exposure } \\
(\mathrm{s})\end{array}$ \\
\hline lckg01010 & G130M/1291 & $2016-01-04$ & $03: 06: 56$ & 450 \\
lckg01020 & G130M/1318 & $2016-01-04$ & $03: 17: 49$ & 440 \\
lckg01030 & G130M/1327 & $2016-01-04$ & $03: 28: 26$ & 880 \\
lckg01040 & G160M/1611 & $2016-01-04$ & $04: 35: 27$ & 800 \\
lckg01050 & G160M/1623 & $2016-01-04$ & $05: 04: 41$ & 800 \\
lckg01060 & G140L/1280 & $2016-01-04$ & $06: 10: 51$ & 2320 \\
lckg01070 & G140L/1280 & $2016-01-07$ & $01: 07: 30$ & 1740 \\
lckg02010 & G140L/1280 & $2016-01-07$ & $02: 43: 57$ & 5161 \\
\hline
\end{tabular}

lifetime position 3. The wavelength zero points of all spectra were adjusted after processing by measuring the wavelengths of strong interstellar features and aligning them to the line-ofsight velocity for $\mathrm{H}$ I, $V_{L S R}=-11 \mathrm{~km} \mathrm{~s}^{-1}$ (Murphy et al. 1996). Improvements in the COS wavelength calibration now give relative uncertainties of $\sim 5 \mathrm{~km} \mathrm{~s}^{-1}$. Comparing the eight exposures in Table 4, fluxes in the wavelength regions each has in common agree to better than $2 \%$, and within the statistical errors of each exposure. Therefore, we combine all exposures for each grating to make three separate spectra for G130M, G160M, and G140L, and we join G130M and G160M at $1423 \AA$ to make a single high resolution spectrum covering 1135$1800 \AA$. Figure 10 shows the full merged COS spectrum from the Lyman limit to $2000 \AA$.

\subsection{Measuring the Broad UV Absorption Lines}

Unlike our discovery of broad C IV absorption in the 2009 and 2010 COS spectra of Mrk 335 (Longinotti et al. 2013), in our new observation we know where to look to see if the absorption features have reappeared in our triggered observations of Mrk 335 in an obscured state. Upon close inspection, broad absorption in C IV and Ly $\alpha$ were immediately apparent in our 2016 observations. To measure the properties of these absorption features, we first developed a total emission model for Mrk 335 that includes both the continuum and the emission lines. As in Longinotti et al. (2013), we use a power law for the continuum, $\mathrm{F}_{\lambda}=\mathrm{F}_{1000}(\lambda / 1000 \AA)^{-\alpha}$ reddened by $\mathrm{E}(\mathrm{B}-$ $\mathrm{V})=0.030$ (Schlafly \& Finkbeiner 2011) using the Cardelli et al. (1989) extinction law with $\mathrm{R}_{\mathrm{V}}=3.1$. Our model includes more components than in Longinotti et al. (2013), as we include components for weaker emission lines as well as Si IV, and the C III $\lambda 977$, N III $\lambda 991$, Si III] $\lambda 1892$, and C III] $\lambda 1909$ lines present in the the G140L spectrum.

We fit our model in three separate pieces due to the complexity of the spectrum and the greatly differing resolutions of the G130M+G160M and the G140L gratings. We first fit G130M+G160M, covering 1140-1800 $\AA$ as described below, then separately fit the Si III] $\lambda 1892$, and C III] $\lambda 1909$ region at the red end of G140L, and the $\mathrm{Ly} \beta+\mathrm{O}$ VI region on the blue end. For the G140L fits we constrain the power-law continuum to have the same spectral index as in the fit to the G130M+G160M spectrum, but we allow its normalization to adjust to the slight $(\sim 2 \%)$ differences relative to G130M and G160M.

In Longinotti et al. (2013), the restricted wavelength range of the G130M and G160M spectra did not include a substantial contribution from the forest of Fe II emission lines that blend into a pseudo-continuum starting at roughly $1500 \AA$. This pseudo-continuum is brighter in our current G140L spectrum, but there are no definitive spectral features that enable us to constrain its strength. These lie mostly at longer wavelengths, peaking at $\sim 2500$ $\AA$. We therefore used the FOS spectrum described in Longinotti et al. (2013) to constrain its normalization, and included it as a fixed element of our model, using a scaled version of the Wills et al. (1985) model convolved with a Gaussian full-width at half maximum (FWHM) of $2800 \mathrm{~km} \mathrm{~s}^{-1}$, approximately the FWHM of the C IV emission line. At $1800 \AA$, its flux is 


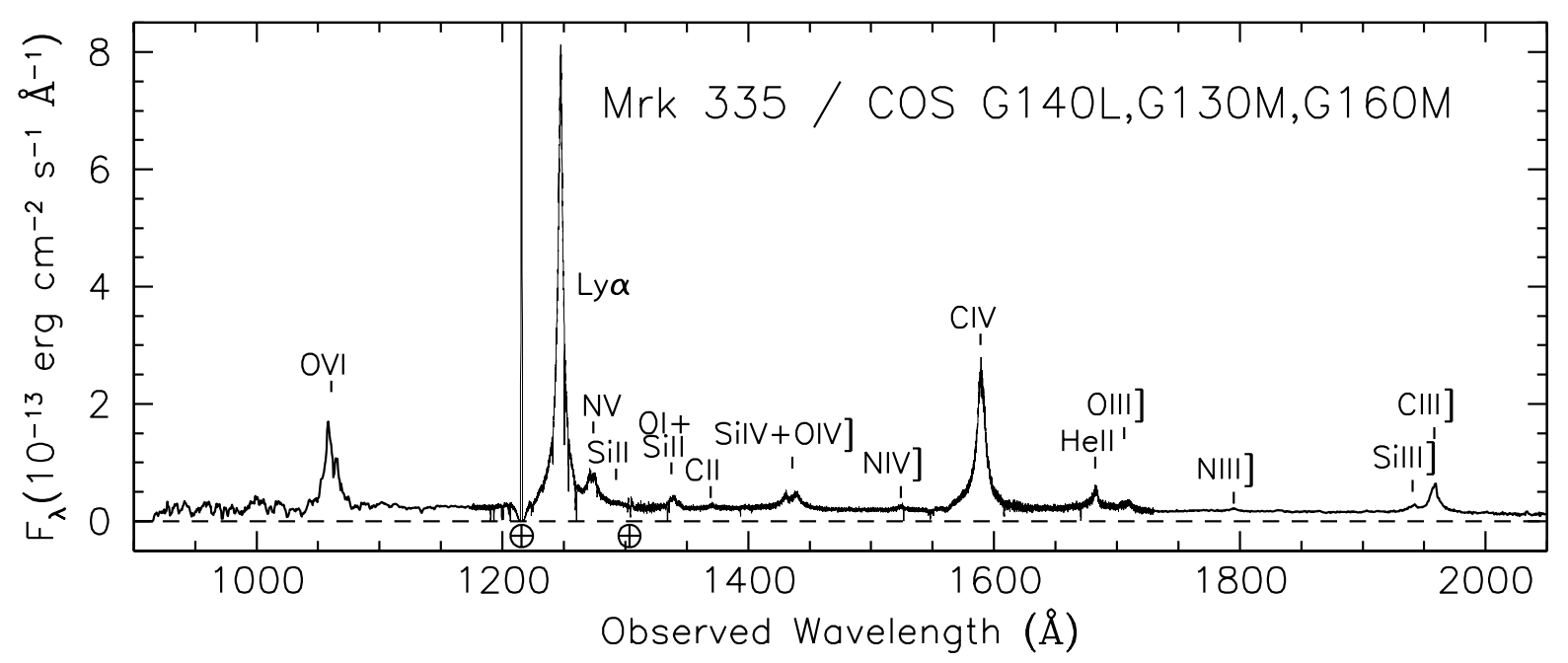

Figure 10. Calibrated COS spectra of Mrk 335 from HST observations on 2016-01-04 and 2016-01-07. Wavelengths shortward of $1180 \AA$ and longward of $1780 \AA$ are from the merged G140L observations. Between $1180 \AA$ and $1423 \AA$, the data are from G130M. Between $1423 \AA$ and $1780 \AA$, the data are from G160M. We mark the prominent emission lines with their identifications. Earth symbols mark geocoronal emission from Ly $\alpha$ and O I $\lambda 1302$.

$3 \times 10^{-16} \operatorname{ergs~cm}^{-2} \mathrm{~s}^{-1} \AA^{-1}$, which is only $2 \%$ of the modeled continuum in our spectrum.

We use multiple Gaussian components for each line, choosing an appropriate number to obtain the best fit for each line. These components have only rough physical meanings. We presume that the narrow components may be more reflective of the narrow-line region (NLR) in Mrk 335, but we ascribe no significance to the broader components we have used to decompose each profile. We are merely interested in a good characterization of the total emission, which will enable us to normalize the spectrum, measure the strengths of the absorption features, and measure the total flux of the emission com- ponents above the continuum. The strongest emission lines in Mrk 335 require at least three, and sometimes four components. The narrowest portions of the profiles are clearly separated in O VI, N V, Si IV, and C IV, so we include a separate component for each line of the doublet. We link the wavelengths of the doublet lines at the ratio of their vacuum wavelengths, and we fix the ratio of their fluxes at a ratio of $2: 1$, assuming they are optically thin. Ly $\alpha$, $\mathrm{N} \mathrm{V}$, Si IV, C IV, and He II all require a very broad Gaussian component with full-width at half maximum (FWHM) 10000 $\mathrm{km} \mathrm{s}^{-1}$. We do not model this separately for the doublets in N V, Si IV, and C IV.

Table 5. Emission Features in the 2016 COS Spectra of Mrk 335.

Parameters with error bars of zero were tied to other parameters in the fit (see 3.2).

\begin{tabular}{ccccc}
\hline \hline Feature & $\begin{array}{c}\lambda_{0} \\
(\AA)\end{array}$ & $\begin{array}{c}\text { Flux } \\
\left(10^{-14} \mathrm{erg} \mathrm{cm}^{-2} \mathrm{~s}^{-1} \AA-1\right)\end{array}$ & $\begin{array}{c}\text { v sys } \\
\left(\mathrm{km} \mathrm{s}^{-1}\right)\end{array}$ & $\begin{array}{c}\text { FWHM } \\
\left(\mathrm{km} \mathrm{s}^{-1}\right)\end{array}$ \\
\hline \hline C III & 977.02 & $13.9 \pm 5.9$ & $-513 \pm 82$ & $3271 \pm 143$ \\
\hline \multicolumn{5}{c}{- Continued on next page } \\
\hline
\end{tabular}


Table 5 - continued from previous page

\begin{tabular}{|c|c|c|c|c|}
\hline Feature & $\begin{array}{l}\lambda_{0} \\
(\AA)\end{array}$ & $\begin{array}{c}\text { Flux } \\
\left(10^{-14} \mathrm{erg} \mathrm{cm}^{-2} \mathrm{~s}^{-1} \AA-1\right)\end{array}$ & $\begin{array}{c}\mathrm{v}_{\mathrm{sys}} \\
\left(\mathrm{km} \mathrm{s}^{-1}\right)\end{array}$ & $\begin{array}{l}\text { FWHM } \\
\left(\mathrm{km} \mathrm{s}^{-1}\right)\end{array}$ \\
\hline N III & 990.82 & $7.5 \pm 5.5$ & $-197 \pm 101$ & $3000 \pm 170$ \\
\hline $\mathrm{O} \mathrm{VI}$ & 1031.93 & $3.9 \pm 7.3$ & $-114 \pm 57$ & $700 \pm 90$ \\
\hline $\mathrm{OVI}$ & 1037.62 & $4.8 \pm 1.4$ & $-113 \pm 0$ & $700 \pm 0$ \\
\hline O VI & 1031.93 & $40.7 \pm 2.1$ & $-38 \pm 0$ & $1193 \pm 130$ \\
\hline $\mathrm{O} \mathrm{VI}$ & 1037.62 & $34.2 \pm 3.4$ & $-37 \pm 0$ & $1193 \pm 0$ \\
\hline $\mathrm{O} \mathrm{VI}$ & 1031.93 & $29.1 \pm 0.1$ & $-123 \pm 0$ & $6210 \pm 220$ \\
\hline $\mathrm{O} \mathrm{VI}$ & 1037.62 & $29.1 \pm 0.0$ & $-125 \pm 0$ & $6210 \pm 0$ \\
\hline $\mathrm{O} \mathrm{VI}$ & 1034.78 & $50.2 \pm 0.0$ & $-130 \pm 0$ & $8736 \pm 0$ \\
\hline S IV & 1072.97 & $4.3 \pm 1.3$ & $234 \pm 120$ & $2900 \pm 180$ \\
\hline $\mathrm{P}$ V & 1117.98 & $1.7 \pm 1.0$ & $1 \pm 134$ & $4393 \pm 0$ \\
\hline $\mathrm{P}$ V & 1128.01 & $1.0 \pm 1.0$ & $1 \pm 0$ & $4393 \pm 0$ \\
\hline C III* & 1176.01 & $0.4 \pm 1.1$ & $-3 \pm 49$ & $1334 \pm 96$ \\
\hline C III* & 1176.01 & $13.6 \pm 2.2$ & $-3 \pm 45$ & $4393 \pm 160$ \\
\hline $\operatorname{Ly} \alpha$ & 1215.67 & $104.0 \pm 2.0$ & $92 \pm 5$ & $687 \pm 10$ \\
\hline $\operatorname{Ly} \alpha$ & 1215.67 & $219.0 \pm 4.1$ & $54 \pm 5$ & $1724 \pm 20$ \\
\hline $\operatorname{Ly} \alpha$ & 1215.67 & $194.0 \pm 4.2$ & $112 \pm 5$ & $4892 \pm 118$ \\
\hline $\operatorname{Ly} \alpha$ & 1215.67 & $78.3 \pm 2.3$ & $92 \pm 14$ & $10743 \pm 196$ \\
\hline N V & 1238.82 & $4.2 \pm 1.1$ & $25 \pm 5$ & $702 \pm 11$ \\
\hline N V & 1242.80 & $4.2 \pm 0.0$ & $24 \pm 0$ & $702 \pm 0$ \\
\hline N V & 1238.82 & $18.6 \pm 2.0$ & $56 \pm 5$ & $2530 \pm 95$ \\
\hline N V & 1242.80 & $18.6 \pm 0.0$ & $57 \pm 0$ & $2530 \pm 0$ \\
\hline N V & 1240.89 & $53.0 \pm 1.2$ & $27 \pm 18$ & $8715 \pm 115$ \\
\hline Si II & 1260.42 & $3.0 \pm 1.0$ & $574 \pm 41$ & $1724 \pm 0$ \\
\hline $\mathrm{O}$ I+Si II & 1304.46 & $1.4 \pm 1.1$ & $-420 \pm 5$ & $800 \pm 133$ \\
\hline $\mathrm{O}$ I+Si II & 1304.46 & $14.5 \pm 1.2$ & $-21 \pm 5$ & $3000 \pm 113$ \\
\hline C II & 1334.53 & $1.3 \pm 1.0$ & $313 \pm 16$ & $800 \pm 0$ \\
\hline C II & 1334.53 & $5.0 \pm 1.0$ & $109 \pm 8$ & $3000 \pm 0$ \\
\hline Si IV & 1393.76 & $4.7 \pm 1.1$ & $110 \pm 13$ & $1021 \pm 112$ \\
\hline Si IV & 1402.77 & $4.7 \pm 0.0$ & $110 \pm 0$ & $1021 \pm 0$ \\
\hline Si IV & 1393.76 & $6.6 \pm 1.1$ & $-61 \pm 14$ & $3645 \pm 135$ \\
\hline Si IV & 1402.77 & $6.6 \pm 0.0$ & $-63 \pm 0$ & $3645 \pm 0$ \\
\hline \multicolumn{5}{|c|}{ - Continued on next page } \\
\hline
\end{tabular}


Table 5 - continued from previous page

\begin{tabular}{|c|c|c|c|c|}
\hline Feature & $\begin{array}{l}\lambda_{0} \\
(\AA)\end{array}$ & $\begin{array}{c}\text { Flux } \\
\left(10^{-14} \mathrm{erg} \mathrm{cm}^{-2} \mathrm{~s}^{-1} \AA-1\right)\end{array}$ & $\begin{array}{c}\mathrm{v}_{\mathrm{sys}} \\
\left(\mathrm{km} \mathrm{s}^{-1}\right)\end{array}$ & $\begin{array}{l}\text { FWHM } \\
\left(\mathrm{km} \mathrm{s}^{-1}\right)\end{array}$ \\
\hline Si IV & 1398.19 & $24.3 \pm 1.1$ & $-1045 \pm 15$ & $10413 \pm 149$ \\
\hline O IV] & 1401.16 & $1.9 \pm 1.1$ & $253 \pm 92$ & $1021 \pm 0$ \\
\hline O IV] & 1401.16 & $9.1 \pm 1.3$ & $253 \pm 0$ & $3645 \pm 0$ \\
\hline N IV] & 1486.50 & $2.1 \pm 1.1$ & $13 \pm 12$ & $1021 \pm 0$ \\
\hline N IV] & 1486.50 & $3.7 \pm 1.0$ & $-84 \pm 17$ & $2590 \pm 154$ \\
\hline C IV & 1548.19 & $30.8 \pm 2.0$ & $39 \pm 5$ & $811 \pm 22$ \\
\hline C IV & 1550.77 & $30.8 \pm 0.0$ & $38 \pm 0$ & $811 \pm 0$ \\
\hline C IV & 1548.19 & $39.6 \pm 3.0$ & $56 \pm 5$ & $1904 \pm 112$ \\
\hline C IV & 1550.77 & $39.6 \pm 0.0$ & $55 \pm 0$ & $1904 \pm 0$ \\
\hline C IV & 1548.19 & $43.1 \pm 3.0$ & $-360 \pm 8$ & $4294 \pm 113$ \\
\hline C IV & 1550.77 & $43.1 \pm 0.0$ & $-360 \pm 0$ & $4294 \pm 0$ \\
\hline C IV & 1549.05 & $74.4 \pm 3.1$ & $56 \pm 14$ & $8736 \pm 119$ \\
\hline He II & 1640.48 & $8.9 \pm 1.1$ & $5 \pm 5$ & $779 \pm 16$ \\
\hline He II & 1640.48 & $8.8 \pm 1.1$ & $-200 \pm 5$ & $2272 \pm 115$ \\
\hline He II & 1640.48 & $57.1 \pm 2.0$ & $-1373 \pm 22$ & $11989 \pm 288$ \\
\hline O III] & 1660.81 & $3.0 \pm 1.0$ & $64 \pm 5$ & $1600 \pm 43$ \\
\hline O III] & 1666.15 & $8.0 \pm 1.0$ & $64 \pm 0$ & $1600 \pm 0$ \\
\hline N III] & 1750.00 & $1.1 \pm 1.0$ & $-1 \pm 68$ & $3267 \pm 324$ \\
\hline Si III] & 1892.08 & $6.1 \pm 0.9$ & $67 \pm 70$ & $1169 \pm 161$ \\
\hline Si III] & 1892.08 & $5.6 \pm 2.0$ & $-635 \pm 541$ & $2920 \pm 121$ \\
\hline C III] & 1908.73 & $16.3 \pm 3.4$ & $12 \pm 19$ & $850 \pm 92$ \\
\hline C III] & 1908.73 & $31.6 \pm 0.9$ & $102 \pm 56$ & $2677 \pm 506$ \\
\hline
\end{tabular}

To optimize our fits, we used the $\mathrm{IRAF}^{2}$ task SPECFIT (Kriss 1994). We first fit the merged G130M+G160M spectra. Our best-fit model has a power-law normalization of $\mathrm{F}_{1000}=4.09 \times$ $10^{-14} \operatorname{ergs~cm}^{-2} \mathrm{~s}^{-1} \AA^{-1}$ with a spectral index

${ }^{2}$ IRAF (http://iraf.noao.edu/) is distributed by the National Optical Astronomy Observatory, which is operated by the Association of Universities for Research in Astronomy, Inc., under cooperative agreement with the National Science Foundation. of $\alpha=1.31$. The fit is excellent, with $\chi^{2}=$ 12049.76 for 12285 points and 99 free parameters. The best fit parameters are given in Table 5. Error bars are calculated from the error matrix of the fit, assuming a $1 \sigma$ error corresponds to $\Delta \chi^{2}=1$ for a single interesting parameter. Parameters with error bars of zero were tied to other parameters in the fit, e.g., the fluxes, wavelengths, and widths of doublet emission lines, and the widths of some of the weakest emission components. 
Strong, broad absorption in Ly $\alpha$ and C IV is clearly present in our new observations. Figure 11 shows our best fit to the $\mathrm{C}$ IV region. Absorption is clearly present on the extreme blue wing of the C IV emission profile (in the same location it appeared in the 2009 COS spectrum). Weak absorption extends all the way down to $\sim 1545 \AA$, past the complex of foreground C IV absorption lines from the Milky Way and the Magellanic stream.

The spectrum and the best-fit emission model for the Ly $\alpha$ region is shown in Figure 11. Unfortunately, absorption corresponding to $\mathrm{N} \mathrm{V}$ falls directly on the peak and the red side of the steep Ly $\alpha$ emission profile. Our model of the Ly $\alpha$ emission is perfectly acceptable without any $\mathrm{N}$ V absorption. Alternatively, if we include N V absorption as Gaussian absorption features approximating the shape of the C IV absorption shown in Figure 11, we can obtain an equally good fit. The parameters of the emission line need only adjust slightly to accommodate the forced inclusion of the absorption features, so it is impossible to get a reliable assessment of any $\mathrm{N} \mathrm{V}$ absorption, although it is likely present.

There is no absorption visible near Si IV, nor near the lower ionization transitions of C III* $\lambda 1176$ or C II $\lambda 1334$. Si IV absorption is visible in the obscuration events seen in NGC 5548 (Kaastra et al. 2014), NGC 985 (Ebrero et al. 2016), and NGC 3783 (Mehdipour et al. 2017), but it was not present in Mrk 335 in 2009. Broad C III* $\lambda 1176$ and C II $\lambda 1334$ absorption (and other lower-ionization species) were present in NGC 5548, and broad C III* $\lambda 1176$ absorption was present in NGC 985, Given this wide array of ionization states seen in the obscured states of other AGN, we measured upper limits for these lines in our current spectrum of Mrk 335 to provide quantitative constraints on the ionization state of the absorbing gas.

\subsubsection{Interstellar absorption in FUSE data}
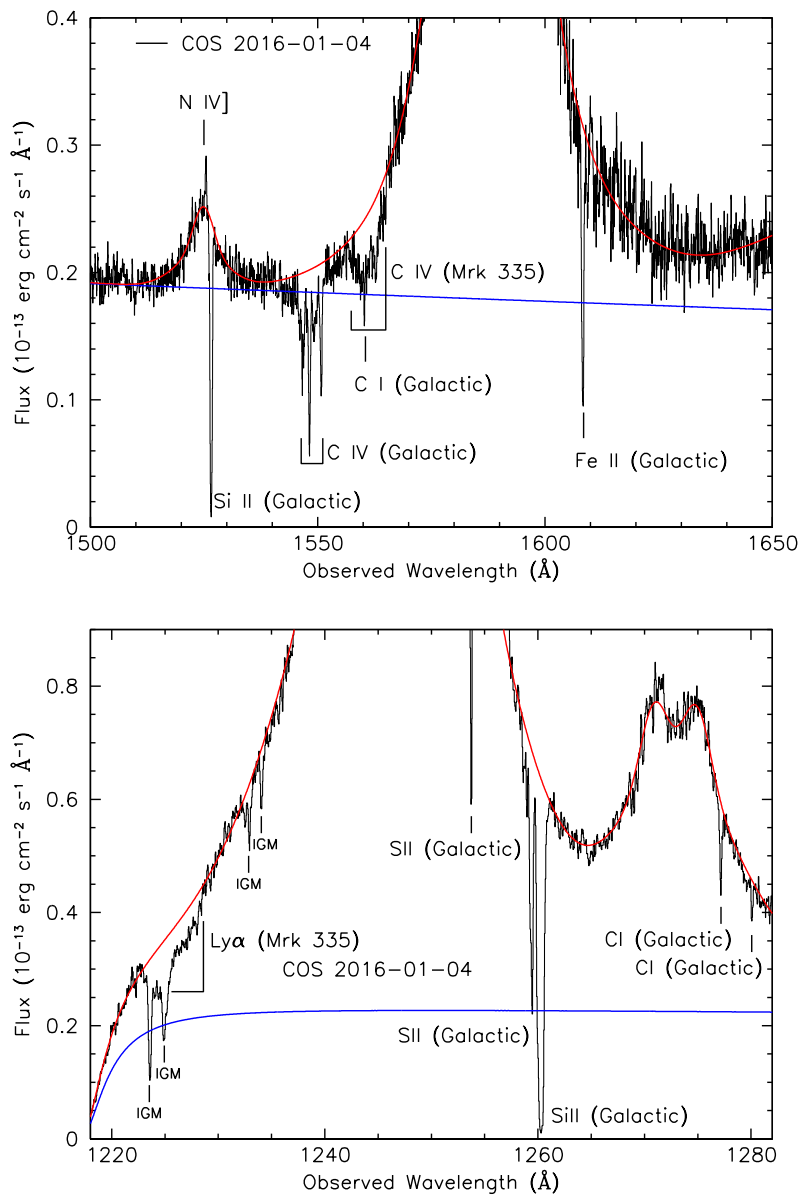

Figure 11. Top: Best fit to the COS G160M 2016 spectrum of the C IV region binned by 4 pixels (black histogram). Bottom: Best fit to the COS G130M 2016 spectrum of the Ly $\alpha+\mathrm{N}$ V region binned by 4 pixels (black histogram). The peak of the C IV (top) and Ly $\alpha$ (bottom) emission lines has been truncated to show more detail at the flux levels of the blue-shifted broad absorption. The solid red line tracing the data is the total emission model. The continuum component is the solid blue line (including foreground damped Milky Way Ly $\alpha$ absorption). Foreground interstellar absorption lines and absorption due to the intergalactic medium (IGM) are marked. The intrinsic broad $\mathrm{C}$ IV ( $\operatorname{Ly} \alpha)$ absorption is marked as the depression below the emission model (at $1227 \AA$ ) surrounding the narrow Galactic C I absorption line.

We now turn to the analysis of the $\mathrm{Ly} \beta+\mathrm{O}$ VI region in the G140L spectrum. The rich foreground ISM absorption at wavelengths short- 
ward of $1100 \AA$ makes this region difficult to model, particularly given the much lower resolution of the G140L grating. However, as a guide we have retrieved a prior high $\mathrm{S} / \mathrm{N}$ spectrum of Mrk 335 from the Mikulski Archive for Space Telescopes (MAST) obtained in 1999 with the Far Ultraviolet Spectroscopic Explorer (FUSE). This makes the modeling more tractable since the FUSE spectrum can provide a very accurate model for all the foreground absorption.

We start by fitting an emission model to the FUSE spectrum of Mrk 335 using our fit to the $\mathrm{C}$ IV profile as a guide. We add components for C III $\lambda$ 977, N III $\lambda 991$, the S IV $\lambda \lambda 1064,1072$ doublets beyond O VI, and the He II/N II $\lambda 1085$ blend. The continuum power law index is fixed at the shape of the COS G130M+G160M spectrum (from 1135 to $1800 \AA$ ), $\alpha=1.31$. We model the foreground ISM absorption using the FUSE spectral simulation tool fsim (V5.0, W. R. Oegerle \& E. M. Murphy). This model matches the location of every single absorption feature in the FUSE spectrum, but it is not completely correct for all the line strengths.

This is convincing evidence that there was no prior intrinsic absorption visible in these earlier high-resolution spectra of Mrk 335. Remarkably, the FUSE data of 1999 provide the ultraviolet view of Mrk 335 nuclear emission during the X-ray bright state, prior to 2007.

This ISM model allows us to fit the emission spectrum (lines+continuum) of Mrk 335 as observed with FUSE very well. We then use this fit to produce a normalized spectrum of Mrk 335. Since all emission is described by our model, and we have identified all absorption features with foreground ISM features (metal lines plus $\mathrm{H}_{2}$ ), this normalized spectrum represents the transmission spectrum of the ISM along the line of sight to Mrk 335.. All features in this spectrum are foreground ISM absorption, and an independent model of the ISM absorption (which was not quantitatively accurate) is no longer

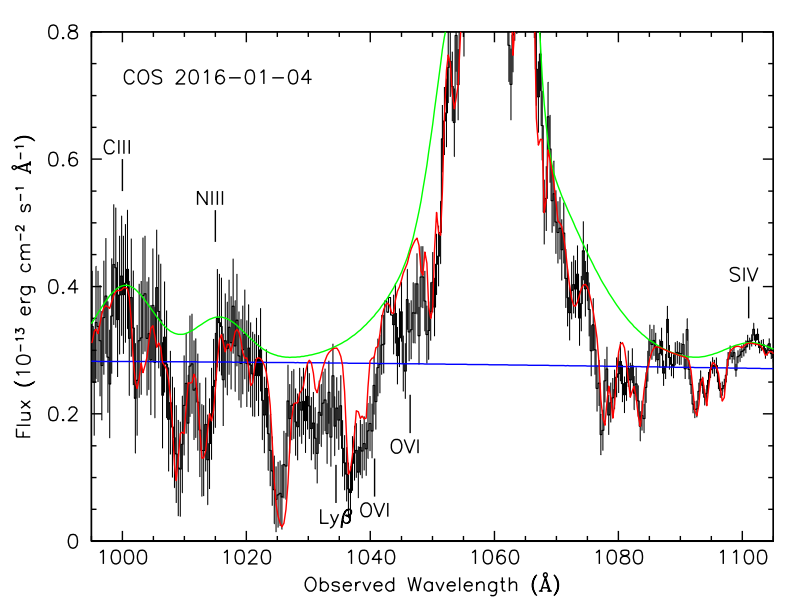

Figure 12. Best fit to the COS G140L 2016 spectrum of the O VI region binned by 4 pixels (black histogram). The peak of the O VI emission line has been truncated to show more detail at the flux levels of the blue-shifted broad absorption in O VI and Ly $\beta$. The solid green line shows the total emission model. The solid red line tracing the data is the total emission model modified by our model of the foreground interstellar absorption. The continuum component is the solid blue line. Emission lines in Mrk 335 and foreground interstellar absorption lines are marked. The intrinsic broad absorption troughs in O VI and Ly $\beta$ are marked as the depressions below the total model on the extreme blue wing of the O VI emission line.

required. We then convolve this transmission spectrum with the COS G140L line spread function to produce a model of the ISM transmission as it would appear in the COS spectrum.

\subsubsection{Interstellar absorption in COS data}

Given this model for the complex, contaminating foreground absorption we can now fit an emission model to the COS G140L spectrum of Mrk 335. To test for the presence of O VI and Ly $\beta$ absorption, we first fit the entire region with just emission components. The weak lines of C III $\lambda 977, \mathrm{~N}$ III $\lambda 991$, and S IV $\lambda 1072$ all require only a single Gaussian component. For O VI, we require the narrow, semi-broad, and broad components for each member of the doublet as in $\mathrm{C}$ IV, but no very broad component 
Table 6. Statistics of Fits to the $\mathrm{Ly} \beta+\mathrm{O}$ VI Region of Mrk 335

\begin{tabular}{lccrc}
\hline \hline Model & $\chi^{2}$ & $\Delta \mathrm{N}_{\text {par }}$ & $\Delta \chi^{2} / \chi_{\nu}^{2}$ & $\mathrm{P}\left(\Delta \chi^{2}\right)$ \\
& & & & \\
\hline \hline No absorption & 541.51 & 0 & 0.0 & $\ldots$ \\
Add Ly $\beta$ only & 518.75 & 1 & 16.22 & $<0.001$ \\
Add O VI only & 532.58 & 2 & 6.36 & 0.002 \\
Add Ly $\beta+\mathrm{O}$ VI & 509.32 & 3 & 22.95 & $<0.001$ \\
\hline
\end{tabular}

is necessary. We constrain the FWHM of the two broad components of the O VI emissionline profile to match those of $\mathrm{C} I V$, but allow the strengths and positions to vary freely. Our best fit gives $\chi^{2}=541.51$ for 400 points and 14 free parameters. This fit is illustrated in Figure 12. All narrow absorption features, which correspond to foreground Galactic ISM absorption, are well matched except in the regions we expect to be affected by broad Ly $\beta$ and O VI absorption.

One can see that Ly $\beta$ and O VI absorption appear to be present in our spectrum, but we test for this more quantitatively by adding in broad Gaussian absorption components for $\mathrm{Ly} \beta$ and O VI based on the locations and shapes of the Ly $\alpha$ and C IV troughs. We test the significance of adding these components individually and severally, as summarized in Table 6 . Including absorption components for all three lines, Ly $\beta$, O VI $\lambda 1031$ and O VI $\lambda 1037$ gives an improvement in $\chi^{2}$ of $\Delta \chi^{2}=32.19$. For an $F$ test with 3 added free parameters, this is a significant improvement at greater than $99.9 \%$ confidence.

With our emission-model fits to the Mrk 335 spectra, we can now divide these into the data to generate normalized spectra. Figure 13 shows normalized spectra in the C IV, Ly $\alpha$, and O VI regions of the Mrk 335 spectrum illustrating the broad absorption features. We also show the (coincident) locations of the X-ray absorp- tion components identified in Longinotti et al. (2013) and in the present paper.

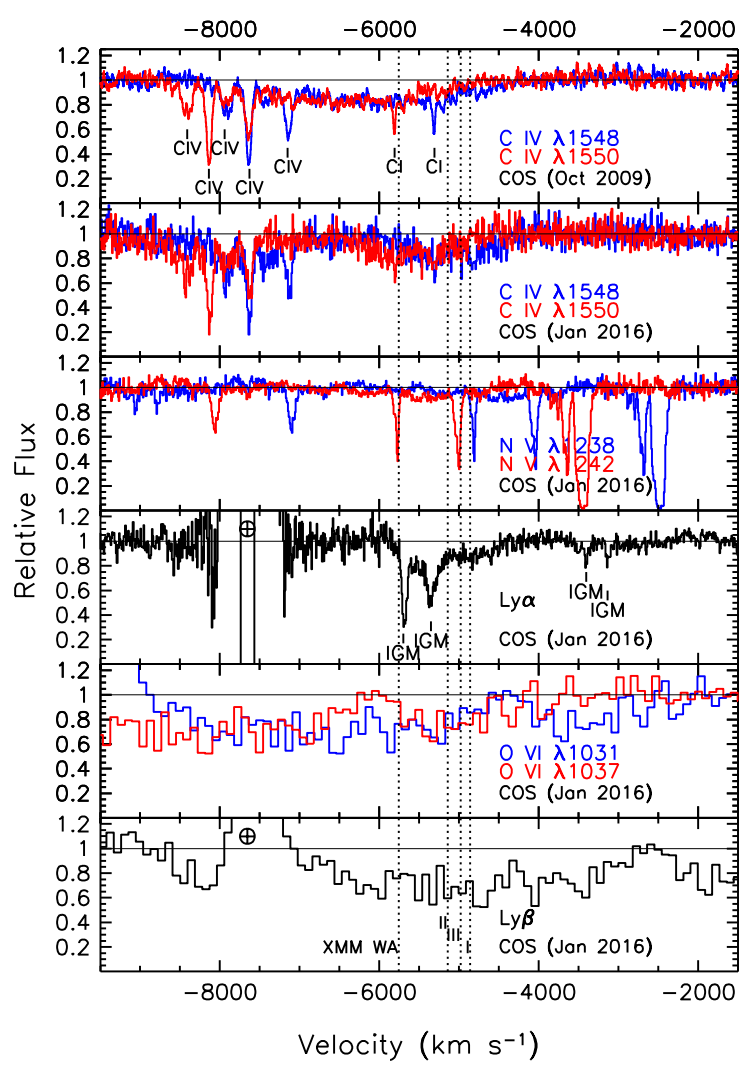

Figure 13. Normalized spectra of Mrk 335 showing relative fluxes vs. velocity relative to the systemic redshift of $z=0.025785$ (Huchra et al. 1999). Top panel: COS spectrum of the C IV region from 2009-10-31. Second panel: COS spectrum from this campaign, 2016-01-04. Red curves give velocities relative to the red component of the doublet, and blue curves are relative to the blue component of the doublet. From third to bottom panel: Ly $\alpha$, $\mathrm{O}$ VI and $\operatorname{Ly} \beta$ regions. Dotted vertical lines indicate X-ray absorption velocities (from this work and from Longinotti et al. (2013)). The narrow absorption features in each panel are foreground Galactic or Intergalactic (IGM) absorption lines.

We measured the strengths of the absorption features in our spectra of Mrk 335 by directly integrating the normalized spectra shown in Figure 13 . We chose velocity intervals covering the 
Table 7. Wavelength Intervals for Mrk 335 Broad Absorption Features

\begin{tabular}{lccccc}
\hline \hline Feature & $\begin{array}{c}\lambda_{\text {vac }} \\
(\AA)\end{array}$ & $\begin{array}{c}v_{1} \\
\left.\mathrm{~km} \mathrm{~s}^{-1}\right)\end{array}$ & $\begin{array}{c}v_{2} \\
\left(\mathrm{~km} \mathrm{~s}^{-1}\right)\end{array}$ & $\begin{array}{c}\lambda_{1} \\
(\AA)\end{array}$ & $\begin{array}{c}\lambda_{2} \\
(\AA)\end{array}$ \\
\hline Ly $\beta$ & 1025.72 & -5976 & -4353 & 1031.4 & 1037.0 \\
O vI & 1031.93 & -5989 & -4375 & 1037.6 & 1043.2 \\
O vI & 1037.62 & -5966 & -4648 & 1043.4 & 1048.0 \\
Ly $\alpha$ & 1215.67 & -5976 & -4362 & 1222.4 & 1229.0 \\
C IV & 1549.05 & -8977 & -4542 & 1542.1 & 1565.1 \\
\hline
\end{tabular}

full range of absorption visible by inspection. Note that this interval is substantially larger for the blended C IV doublet, both because the two components are separated by $950 \mathrm{~km} \mathrm{~s}^{-1}$ in velocity space, but also because there is no confusion on the blue wing of the absorber. The corresponding blue end in Ly $\alpha$ is buried under the damped Ly $\alpha$ absorption of the Milky Way. Likewise, Ly $\beta$ is contaminated by geocoronal Ly $\beta$ emission. For O VI, the doublet separation is $1814 \mathrm{~km} \mathrm{~s}^{-1}$, causing blending at higher blue-shifted velocities. We therefore limited the range for integration to approximately the same interval used for Ly $\alpha$. All features have broad widths that are well resolved. This enables us to directly integrate the normalized absorption profiles to obtain equivalent widths (EW). Our direct integrations also yield column densities using the apparent optical depth method of Savage \& Sembach (1991). Note that Figure 13 shows that all the troughs have similar depths. Thus, they are likely saturated, and therefore we quote the column densities as lower limits. Given this likelihood of saturation, we also tabulate for each line the covering fraction implied if the deepest part of the trough is saturated. The properties of the absorbers are summarized in Table 8.

\section{DISCUSSION}

The latest X-ray-UV observational campaign carried out on Mrk 335 in 2015 and presented in this work fully confirms the scenario proposed by Longinotti et al. (2013) based on nonsimultaneous data. We briefly recall the properties of the absorber in Mrk 335 reported therein.

\subsection{The warm absorber in 2009 and 2015}

In our comparison with present data we consider only the 2009 spectrum, which in Longinotti et al. (2013) is referred to as the "the mid-state" and in which the warm absorber properties could be measured at best compared to other epochs. The ionization was there described in terms of the ionization parameter $\xi=\mathrm{L} / \mathrm{nr}^{2}$ therefore we now quote the corresponding number in terms of $\mathrm{U}$ to ease comparison with Table 3.

Table 9 reports the values of the three layers of ionized absorption detected in 2009 adapted from Table 5 in Longinotti et al. (2013). The ionized absorber in 2015 (see Table 3) seems very consistent with WA II in 2009. This seems to indicate that since its first record in 2009, the ionized wind has become a persistent feature of Mrk 335, which is also supported by the conclusions reached by Gallo et al. (2018) in their long-term study. These authors conclude that the current low flux state observed since 2007 is not driven by changes in the structure of the inner accretion disc. Rather, they propose that the variability pattern may be explained either in terms of coronal changes or intervening absorption. The apparent stability of the outflow supports therefore the latter hypothesis. We note that in the first low state spectrum of 2007 (Grupe et al. 2007), the presence of ionized absorption could not be investigated in detail due the low $\mathrm{S} / \mathrm{N}$ of the grating data (see Longinotti et al. 2013). However, as the presence of the absorber in this epoch could not be excluded either, we speculate that this wind may well have emerged in 2007 when Mrk 335 entered its prolonged low X-ray flux state. In the following we proceed to explore the possible association of the X-ray absorbers 
Table 8. Properties of the UV Broad Absorption Lines in Mrk 335. Velocities and FWHM of O VI and Ly $\beta$ lines are tied to those of $\mathrm{C} \mathrm{IV}$, whereas for $\mathrm{N}$ V they are tied to Ly $\alpha$ values. Upper limits of C III*, C II, and Si IV were determined with tabulated values.

\begin{tabular}{lcccccc}
\hline \hline Line & $\begin{array}{c}\lambda_{o} \\
(\AA)\end{array}$ & $\begin{array}{c}\text { EW } \\
(\AA)\end{array}$ & $\begin{array}{c}\text { Velocity } \\
\left(\mathrm{km} \mathrm{s}^{-1}\right)\end{array}$ & $\begin{array}{c}\text { FWHM } \\
\left(\mathrm{km} \mathrm{s}^{-1}\right)\end{array}$ & $\mathrm{C}_{\mathrm{f}}$ & $\begin{array}{c}\log \mathrm{N}_{\text {ion }} \\
\left(\log \mathrm{cm}^{-2}\right)\end{array}$ \\
\hline \hline Ly $\beta$ & 1025.72 & $0.91 \pm 0.27$ & -5245 & 807 & $0.29 \pm 0.08$ & $>15.43$ \\
O VI & 1031.93 & $0.97 \pm 0.18$ & -5245 & 807 & $0.29 \pm 0.08$ & $>14.93$ \\
O VI & 1037.62 & $0.93 \pm 0.12$ & -5245 & 807 & $0.27 \pm 0.05$ & $>15.20$ \\
Ly $\alpha$ & 1215.67 & $0.74 \pm 0.03$ & $-5144 \pm 36$ & $685 \pm 74$ & $0.22 \pm 0.01$ & $>14.15$ \\
N V & 1240.81 & $0.08 \pm 0.02$ & $-5144 \pm 36$ & $685 \pm 74$ & $0.05 \pm 0.03$ & $>13.86$ \\
C III* & 1176.01 & $<0.015$ & -5144 & 700 & $\ldots$ & $<12.15$ \\
C II & 1334.53 & $<0.015$ & -5144 & 700 & $\ldots$ & $<12.84$ \\
Si IV & 1393.76 & $<0.017$ & -5144 & 700 & $\ldots$ & $<12.28$ \\
C IV & 1549.05 & $2.17 \pm 0.10$ & $-5245 \pm 16$ & $807 \pm 75$ & $0.19 \pm 0.04$ & $>14.97$ \\
\hline
\end{tabular}

Table 9. Warm absorber properties in the 2009 RGS spectrum (adapted from Longinotti et al. 2013).

\begin{tabular}{ccccc}
\hline Phase & $\begin{array}{c}\text { Log } \mathrm{U} \\
-\end{array}$ & $\begin{array}{c}\log \mathrm{N}_{H} \\
\left(\mathrm{~cm}^{-2}\right)\end{array}$ & $\begin{array}{c}\mathrm{v}_{\text {out }} \\
\left(\mathrm{km} \mathrm{s}^{-1}\right)\end{array}$ & $\begin{array}{c}\mathrm{v}_{\text {broad }} \\
\left(\mathrm{km} \mathrm{s}^{-1}\right)\end{array}$ \\
\hline I & $0.39_{-0.11}^{+0.04}$ & $21.34 \pm 0.06$ & $4000_{-700}^{+180}$ & $\leq 100$ \\
II & $1.04 \pm 0.03$ & $21.63 \pm 0.06$ & $5200_{-60}^{+190}$ & $\leq 100$ \\
III & $2.05_{-0.09}^{+0.05}$ & $22.55 \pm 0.15$ & $5300_{-100}^{+90}$ & $\leq 100$ \\
\hline \hline
\end{tabular}

to the UV wind with the advantage provided by the simultaneity of the two sets of observations in 2015/16.

\subsubsection{X-ray and UV absorbers in 2015: the RGS view}

We start by comparing the parameters estimated by the UV lines with those of the soft X-ray warm absorber for which more precise constraints are available owing to the higher detail provided by the grating spectra. The outflow velocity in both bands shows remarkable coincidence. However, the X-rayestimated ionic column densities provided by the photoionization model of the soft X-ray absorber in Table 3 are only partially com- patible with the columns estimated by the UV throughs (Table 8): this X-ray warm absorber does not produce enough C IV absorption $\left(\mathrm{N}_{C I V}=4.7 \times 10^{12} \mathrm{~cm}^{-2}\right)$ although it might contribute to the O VI $\left(\mathrm{N}_{O V I}=7 \times 10^{15} \mathrm{~cm}^{-2}\right.$ ) and $\mathrm{NV}\left(\mathrm{N}_{N V}=5.38 \times 10^{13} \mathrm{~cm}^{-2}\right)$. This partial discrepancy can be visualized in Figure 14 where we can see that the limits traced by the UV columns intersect the X-ray warm absorber columns only for OVI and NV.

As noted in Section 2.1, the spectral fits to the RGS data do not allow us to constrain the width of the X-ray absorption lines, but they seem to favour the presence of narrow rather than broad absorption lines, which may also poise a problem to interpret the two outflows as arising from the same gas. Nonetheless, if it is postulated that X-ray photons cross a smaller range of velocities compared to UV photons as proposed in the sketch of Figure 15, we may explain why broader absorption lines are detected in the UV band compared to the narrow lines observed in the X-rays. 
Another considerable difference of the wind in the two bands is the covering fraction of the gas. While the coverage of the soft X-ray ionized gas estimated from the RGS is $100 \%$, the UV absorber covers a small fraction of the ionizing continuum (20-30\%. see Table 8$)$. We tested for the presence of a partially covering warm absorber by using a PHASE version with variable covering factor. This test indicates that the warm absorber coverage is consistent with being as low as $80 \%$, although partial covering is not formally required by the fit statistics. We note that such value is fully coincident with the constraint obtained for the warm absorber in the mid state flux of 2009. This may indicate that the hotter part of the outflow (seen in Xrays) is organized in a denser/clumpier structure than the gas ionized by the UV continuum, and/or it may also indicate that the UV source is more extended than the X-ray source, as depicted in Figure 15. This interpretation is compatible and it may actually explain the presence of the partial covering absorber observed in the broadband X-ray spectrum (see next section).

\subsubsection{X-ray and UV absorbers in 2015: the EPIC-pn view}

The 2015 CCD spectrum of Mrk 335 reveals the presence of two additional layers of absorption: the highly ionized absorber described in Section 2.2.2 ( $\mathrm{v}_{\text {out }}=5,200_{-200}^{+700} \mathrm{~km} \mathrm{~s}^{-1}$, $\left.\log \mathrm{U}=3.13_{-0.59}^{+0.09}, \quad \log \mathrm{N}_{H} \geq 23.07\right)$ and the partial covering absorber described in Section 2.2 $\left(\log \left(\mathrm{N}_{H}\right)=22.99 \pm 0.06, \mathrm{C}_{f}=0.79_{-0.05}^{+0.02}\right.$ and $\left.\log \mathrm{U}<1.35\right)$. Whereas the former component is too highly ionized so as to affect the UV spectrum, we explore a possible UV connection with the latter one.

Despite the lack of more detailed properties inferred on the partially covering gas, the $\mathrm{X}$ ray spectral fits show that it has a moderately low ionization, high column density, and that it covers around $80 \%$ of the X-ray source. Figure 7 shows that its ionization $(\log U<1.35)$

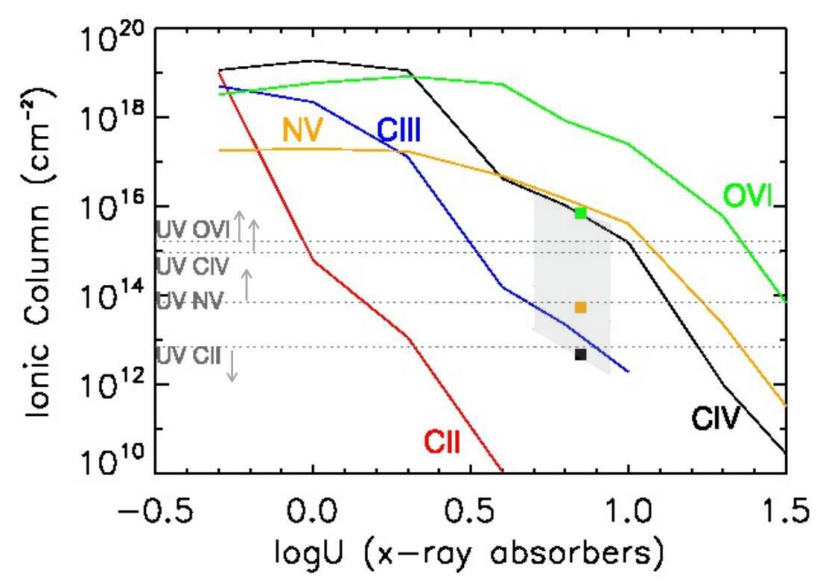

Figure 14. Comparison of the ionic column densities of UV and X-ray absorbers. Solid lines trace the ionic columns for the X-ray partial covering absorber calculated for the best fit column density of $\log \left(\mathrm{N}_{H}\right)=22.99 \pm 0.06$. Squared points and grey shade respectively mark the best fit values of the OVI, NV, CIV column densities (same color code) and the area allowed by the X-ray warm absorber detected in RGS (Table 3). Grey horizontal dotted lines trace the upper and lower limits of the UV lines ionic columns reported in Table 8.

overlaps with that of the RGS warm absorber $\left(\log \mathrm{U}=0.85_{-0.14}^{+0.09}\right)$, and so does the covering fraction, as reported in Section 4.1.1. Unfortunately, the outflow velocity of the partial covering gas could not be measured in CCD data (Sec. 2.2.1), therefore it is difficult to pinpoint a more constrained location. In the spectral fitting, we have tied its velocity to the one of the less dense warm absorber detected in RGS assuming that both are part of the intervening gas that crossed our line of sight during the XMM-Newton observation. This is largely justified by the X-ray history of Mrk 335 which, as reported in Sec. 1, did not show intervening ionized absorption prior to the decrease of X-ray flux (Grupe et al. 2007, 2008) that since 2007 gave rise to the several X-ray campaigns launched on this source.

Moreover, the relatively high velocity measured in the X-ray and also in the UV absorbers 
( $5000-6000 \mathrm{~km} / \mathrm{s}$ ) suggests that the obscuring system is located close to the accretion disk or the inner Broad Line Region, and tend to exclude other possible locations placed farther away (e.g. the inner wall of the torus). These considerations has led us to associate the partial covering and the warm absorber to the same system.

We speculate that the two X-ray absorbers detected in the present work with such a wide range of column densities but overlapping ionization state and coverage may well be tracing the same system of gas where denser filaments/clouds are producing the observed spectral curvature in the broadband data whereas less dense parts of the outflow are responsible for imprinting the strong Fe UTA absorption. A gas with these characteristics is expected to imprint detectable features in the UV spectrum, which are not currently seen. We plot the predicted ionic columns of this absorber in Figure 14 for the range of the ionization parameter allowed by the best fit. The X-ray curves for the corresponding UV ions show that a partial covering absorber with $\log \mathrm{U} \sim 0.5-1$ is compatible with the same gas producing both the X-ray and UV absorption. As proposed in the previous paragraph, if we postulate that the X-ray and UV absorbers are distributed with very different coverage (80-100\% versus $20-30 \%$ ), then we may well explain why the strong X-ray partially covering gas does not appear in the UV data (see Fig.15).

\subsection{Conclusions}

The obscuring wind in Mrk 335 shows therefore a very rich ionization structure that extends from the UV broad troughs observed by HST up to the highly ionized transitions in the Fe K band, observed in Mrk 335 for the first time. With exception of the outflow velocity ( $\sim 5200 \mathrm{~km} \mathrm{~s}^{-1}$ in CCD data), the properties of this highly ionized wind $\left(\log \mathrm{U}=3.13_{-0.59}^{+0.09}\right.$ and $\left.\log \mathrm{N}_{H} \geq 23.07\right)$ are reminiscent of ultra-

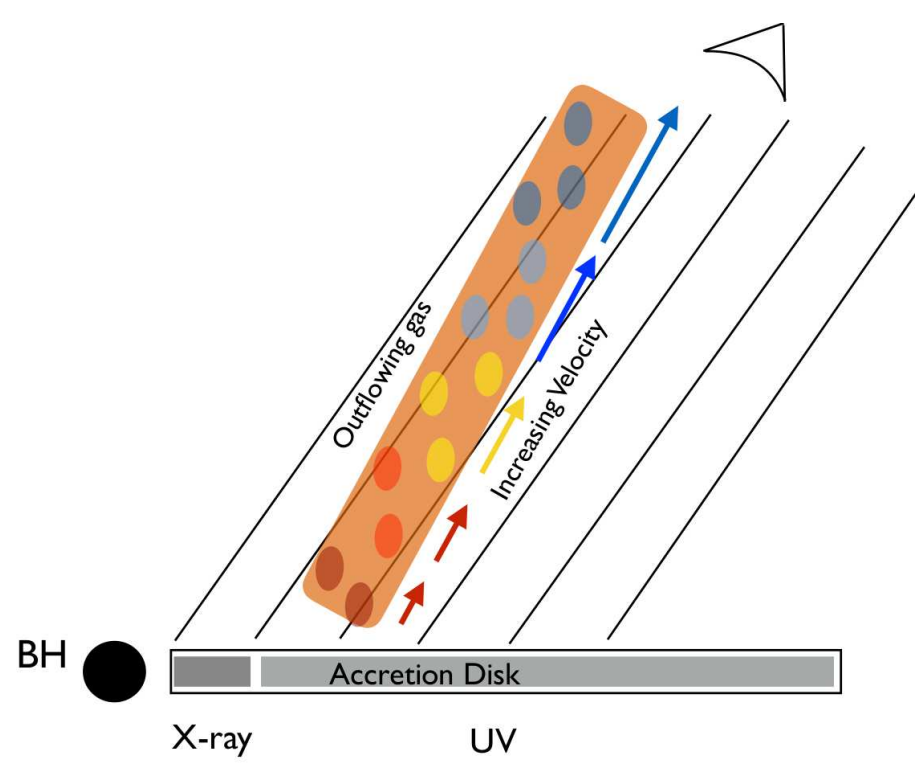

Figure 15. Sketch of the proposed geometry of Mrk 335 outflow. Black lines represent different sight lines. Due to the different sizes of the sources of X-ray and UV photons, the outflowing gas in the line of sight covers almost entirely the X-ray source and only a small fraction of the UV-emitting disk. The outflowing gas is constituted by a distribution of ionized gas responsible for the X-ray ionized absorber(s) and by denser discrete clouds/filaments that produce the partial covering in the continuum. Colors represent the increasing velocity along the line of sight: X-ray photons cross a small range of velocities compared to UV photons, which may explain why UV absorption lines are broader than the narrow lines observed in the X-ray spectrum.

fast-outflows that are seen in Seyfert Galaxies (Tombesi et al. 2011) and whose appearance seems to bear relation with low luminosity states of the sources (Matzeu et al. 2017). The presence of an even faster component of the wind in this low flux state of Mrk 335 cannot be assessed as the EPIC-pn data are heavily affected by high background at $\mathrm{E} \geq 8 \mathrm{keV}$ (but see Gallo et al. 2019, for the analysis of the flaring portion of this data). However, we do not exclude the presence of additional and possibly faster outflow components that may also explain unidentified absorption lines in the RGS spectrum (see Figure 4). 
Longinotti et al. (2013) extensively discussed the possible interpretation for the appearance of the wind and based on the variability of the broad UV absorption troughs, concluded that the outflow was transiting our line of sight to the central source at the scale of the Broad Line Region $\left(0.7-4 \times 10^{16} \mathrm{~cm}\right)$. The present data not only brings a strong evidence on the persistency of the wind, as discussed above, but it also provides a more corroborated association of the UV and X-ray outflows. The absorber therefore can be effectively tracing the base of a radiatively driven wind produced by the accretion disc (Proga \& Kallman 2004) as suggested for sources with similar behavior as NGC 5548 and NGC 3783 (Kaastra et al. 2014; Mehdipour et al. 2017). In Mrk 335 the situation seems to be akin to NGC 5548 where the obscuring gas covers $70 \%$ of the source and where the obscuration is observed to extend for several years compared to the isolated eclipsing event that recently characterized NGC 3783 (Mehdipour et al. 2017).

Further results on the behavior of the absorbers in Mrk 335 are expected by an ongoing multi-epoch study (Parker et al. in prep.) that include data from very recent X-ray/UV campaigns launched in 2018 and 2019.

\section{ACKNOWLEGMENTS}

Based on proprietary observations obtained with the NASA/ESA HST and with XMMNewton, an ESA science mission with instruments and contributions directly funded by ESA Member States and NASA. Some of the data presented in this paper were obtained from the Mikulski Archive for Space Telescopes (MAST). This work was supported by NASA through a grant for $H S T$ program number 13814 from the Space Telescope Science Institute (STScI), which is operated by the Association of Universities for Research in Astronomy, Incorporated, under NASA contract NAS5-26555. This research has made use of the NASA/IPAC Extragalactic Database (NED), which is operated by the Jet Propulsion Laboratory, California Institute of Technology, under contract with the National Aeronautics and Space Administration. YK acknowledges support from DGAPAPAIIPIT grant IN106518. ALL acknowledges support from CONACyT grant CB-2016-01286316.

\section{REFERENCES}

$? ? ? ?$

08. 1

Arnaud, K. A. 1996, in Astronomical Society of the Pacific Conference Series, Vol. 101,

Astronomical Data Analysis Software and Systems V, ed. G. H. Jacoby \& J. Barnes, 17

Bianchi, S., Matt, G., Haardt, F., et al. 2001, AA, 376, 77

Cardelli, J. A., Clayton, G. C., \& Mathis, J. S. 1989, ApJ, 345, 245

Cash, W. 1979, ApJ, 228, 939

Chainakun, P., \& Young, A. J. 2015, MNRAS, 452,333
Crenshaw, D. M., Kraemer, S. B., \& George, I. M. 2003, ARA\&A, 41, 117

Ebrero, J., Kriss, G. A., Kaastra, J. S., \& Ely, J. C. 2016, AA, 586, A72

Feruglio, C., Fiore, F., Carniani, S., et al. 2015, AA, 583, A99

Fukumura, K., Kazanas, D., Contopoulos, I., \& Behar, E. 2010, ApJ, 715, 636

Gallo, L. C., Blue, D. M., Grupe, D., Komossa, S., \& Wilkins, D. R. 2018, MNRAS, 478, 2557

Gallo, L. C., Fabian, A. C., Grupe, D., et al. 2013, MNRAS, 428, 1191

Gallo, L. C., Wilkins, D. R., Bonson, K., et al. 2015, MNRAS, 446, 633 
Gallo, L. C., Gonzalez, A. G., Waddell, S. G. H., et al. 2019, MNRAS, 484, 4287

Green, J. C., Froning, C. S., Osterman, S., et al. 2012, ApJ, 744, 60

Grupe, D., Komossa, S., \& Gallo, L. C. 2007, ApJ, 668, L111

Grupe, D., Komossa, S., Gallo, L. C., et al. 2008, ApJ, 681, 982

—. 2012, ApJS, 199, 28

Grupe, D., Longinotti, A. L., Kriss, G., et al. 2015, The Astronomer's Telegram, 8477

Huchra, J. P., Vogeley, M. S., \& Geller, M. J. 1999, ApJS, 121, 287

Kaastra, J. S., Kriss, G. A., Cappi, M., et al. 2014, Science, 345, 64

Kalberla, P. M. W., Burton, W. B., Hartmann, D., et al. 2005, AA, 440, 775

Kara, E., Fabian, A. C., Cackett, E. M., et al. 2013, MNRAS, 434, 1129

Keek, L., \& Ballantyne, D. R. 2016, MNRAS, 456, 2722

King, A., \& Pounds, K. 2015, ARA\&A, 53, 115

Kinkhabwala, A., Sako, M., Behar, E., et al. 2002, ApJ, 575, 732

Komossa, S., Grupe, D., Schartel, N., et al. 2017, in IAU Symposium, Vol. 324, New Frontiers in Black Hole Astrophysics, ed. A. Gomboc, 168-171

Königl, A., \& Kartje, J. F. 1994, ApJ, 434, 446

Kriss, G. 1994, Astronomical Data Analysis Software and Systems, 3, 437

Kriss, G. A., Mehdipour, M., Kaastra, J. S., et al. 2018, ArXiv e-prints, arXiv:1810.12206

Krolik, J. H., \& Kriss, G. A. 2001, ApJ, 561, 684

Krongold, Y., Nicastro, F., Elvis, M., et al. 2007, ApJ, 659, 1022

Krongold, Y., Elvis, M., Andrade-Velazquez, M., et al. 2010, ApJ, 710, 360

Larsson, J., et al. 2008, MNRAS, 384, 1316

Longinotti, A. L., Nucita, A., Santos-Lleo, M., \& Guainazzi, M. 2008, AA, 484, 311
Longinotti, A. L., Sim, S. A., Nandra, K., \& Cappi, M. 2007, MNRAS, 374, 237

Longinotti, A. L., Krongold, Y., Kriss, G. A., et al. 2013, ApJ, 766, 104

Longinotti, A. L., Vega, O., Krongold, Y., et al. 2018, ApJ, 867, L11

Mathur, S., Elvis, M., \& Wilkes, B. 1995, ApJ, 452,230

Mathur, S., Wilkes, B., \& Elvis, M. 1998, ApJ, 503, L23

Matzeu, G. A., Reeves, J. N., Braito, V., et al. 2017, MNRAS, 472, L15

Mehdipour, M., Kaastra, J. S., Kriss, G. A., et al. 2017, AA, 607, A28

Murphy, E. M., Lockman, F. J., Laor, A., \& Elvis, M. 1996, ApJS, 105, 369

Netzer, H. 2004, ApJ, 604, 551

Ogle, P. M., Marshall, H. L., Lee, J. C., \& Canizares, C. R. 2000, ApJ, 545, L81

O’Neill, P. M., Nandra, K., Cappi, M., Longinotti, A. L., \& Sim, S. A. 2007, MNRAS, 381, L94

Parker, M. L., Wilkins, D. R., Fabian, A. C., et al. 2014, MNRAS, 443, 1723

Proga, D., \& Kallman, T. R. 2004, ApJ, 616, 688

Savage, B. D., \& Sembach, K. R. 1991, ApJ, 379, 245

Schlafly, E. F., \& Finkbeiner, D. P. 2011, ApJ, 737, 103

Tombesi, F., Cappi, M., Reeves, J. N., et al. 2011, ApJ, 742, 44

Tombesi, F., Meléndez, M., Veilleux, S., et al. 2015, Nature, 519, 436

Turner, T. J., Nandra, K., Zdziarski, A. A., et al. 1993, ApJ, 407, 556

Wilkins, D. R., \& Gallo, L. C. 2015, MNRAS, 449, 129

Wilkins, D. R., Gallo, L. C., Grupe, D., et al. 2015, MNRAS, 454, 4440

Wills, B. J., Netzer, H., \& Wills, D. 1985, ApJ, 288, 94

Wilms, J., Allen, A., \& McCray, R. 2000, ApJ, 542, 914 